\title{
Impaired rRNA synthesis triggers homeostatic responses in hippocampal neurons
}

\section{Anna Kiryk ${ }^{1}$, Katharina Sowodniok ${ }^{2}$, Grzegorz Kreiner ${ }^{3}$, Jan Rodriguez-Parkitna ${ }^{4}$, Aynur Sönmez ${ }^{5}$, Tomasz Górkiewicz ${ }^{1,6}$, Holger Bierhoff', Marcin Wawrzyniak ${ }^{1}$, Artur K. Janusz ${ }^{1}$, Birgit Liss ${ }^{8}$, Witold Konopka ${ }^{1}$, Günther Schütz ${ }^{2}$, Leszek Kaczmarek ${ }^{1}$ and Rosanna Parlato ${ }^{2,5,8 *}$}

${ }^{1}$ Nencki Institute of Experimental Biology, Polish Academy of Sciences, Warsaw, Poland

${ }^{2}$ DKFZ-ZMBH Alliance, Department of Molecular Biology of the Cell I, German Cancer Research Center, Heidelberg, Germany

${ }^{3}$ Department of Brain Biochemistry, Institute of Pharmacology, Polish Academy of Sciences, Cracow, Poland

${ }^{4}$ Department of Molecular Neuropharmacology, Institute of Pharmacology, Polish Academy of Sciences, Cracow, Poland

${ }^{5}$ Institute of Anatomy and Cell Biology, University of Heidelberg, Heidelberg, Germany

${ }^{6}$ Department of Biophysics, Warsaw University of Life Sciences-SGGW, Warsaw, Poland

DKFZ-ZMBH Alliance, Department of Molecular Biology of the Cell II, German Cancer Research Center, Heidelberg, Germany

8 Institute of Applied Physiology, University of UIm, UIm, Germany

\section{Edited by:}

Lavinia Alberi, University of Fribourg,

Switzerland

Reviewed by:

Emanuela Santini, New York

University, USA

Alexis M. Stranahan, Georgia Health

Sciences University, USA

*Correspondence:

Rosanna Parlato, Institute of Applied Physiology, University of UIm, Albert Einsteinallee 11, $89081 \mathrm{Ulm}$,

Germany

e-mail: rosanna.parlato@uni-ulm.de
Decreased rRNA synthesis and nucleolar disruption, known as nucleolar stress, are primary signs of cellular stress associated with aging and neurodegenerative disorders. Silencing of rDNA occurs during early stages of Alzheimer's disease (AD) and may play a role in dementia. Moreover, aberrant regulation of the protein synthesis machinery is present in the brain of suicide victims and implicates the epigenetic modulation of rRNA. Recently, we developed unique mouse models characterized by nucleolar stress in neurons. We inhibited RNA polymerase I by genetic ablation of the basal transcription factor TIF-IA in adult hippocampal neurons. Nucleolar stress resulted in progressive neurodegeneration, although with a differential vulnerability within the CA1, CA3, and dentate gyrus (DG). Here, we investigate the consequences of nucleolar stress on learning and memory. The mutant mice show normal performance in the Morris water maze and in other behavioral tests, suggesting the activation of adaptive mechanisms. In fact, we observe a significantly enhanced learning and re-learning corresponding to the initial inhibition of rRNA transcription. This phenomenon is accompanied by aberrant synaptic plasticity. By the analysis of nucleolar function and integrity, we find that the synthesis of rRNA is later restored. Gene expression profiling shows that 36 transcripts are differentially expressed in comparison to the control group in absence of neurodegeneration. Additionally, we observe a significant enrichment of the putative serum response factor (SRF) binding sites in the promoters of the genes with changed expression, indicating potential adaptive mechanisms mediated by the mitogen-activated protein kinase pathway. In the DG a neurogenetic response might compensate the initial molecular deficits. These results underscore the role of nucleolar stress in neuronal homeostasis and open a new ground for therapeutic strategies aiming at preserving neuronal function.

Keywords: rRNA, nucleolus, learning and memory, hippocampus, mTOR, neurogenesis

\section{INTRODUCTION}

Protein synthesis is essential in the consolidation of long-term memory by long-lasting changes of synapses (Cajigas et al., 2010). Neuronal stimulation and prolonged neuronal activity increase protein synthesis by controlling nucleolar number and/or by regulating processing and maturation of rRNA (Jordan et al., 2007).

In line with a crucial role in learning and memory, rRNA transcription is down-regulated during ageing in CA1 and dentate gyrus (DG) hippocampal areas although in CA3 it is increased to compensate the functional loss in DG and CA1. Interestingly, CA1 and DG are especially vulnerable to brain injury and
Alzheimer's disease (AD), supporting the connection between neuronal activity, metabolism, and functional deficits in aged hippocampal neurons (Garcia Moreno et al., 1997). For example, rRNA transcription is decreased in hippocampal neurons after alcohol consumption and deprivation in rats and it is associated with memory impairment (Garcia-Moreno et al., 2001). Recent studies have shown that rRNA transcription is specifically downregulated in the hippocampus of $\mathrm{AD}$ and suicide subjects by epigenetic modifications (McGowan et al., 2008; Pietrzak et al., 2011). Hence, a better understanding of the cellular and molecular strategies adopted to counteract nucleolar stress could pave the way to neuroprotective strategies preserving neuronal function. 
The mammalian target of rapamycin (mTOR) is an evolutionarily conserved serine/threonine kinase and an important regulator of cell growth and protein translation, dependent on cellular metabolic state and stress conditions (Laplante and Sabatini, 2012). mTOR dysregulation is associated with several neurodevelopmental and neurodegenerative disorders making its targets and regulators valuable for therapeutic interventions (Crino, 2011). In agreement with its fundamental role, several feedback mechanisms intervene to control its function. For example in response to permissive growth conditions mTOR regulates protein synthesis by controlling rRNA transcription (Mayer et al., 2004; Grewal et al., 2007). In turn, inhibition of rRNA transcription and disruption of nucleolar integrity down-regulate mTOR activity (Rieker et al., 2011).

To elucidate the role of rRNA transcription and nucleolar activity in learning and memory, we have used a genetic approach to induce nucleolar stress in specific neurons. This method is based on the conditional ablation of the nucleolar transcription factor TIF-IA, crucial for recruiting RNA polymerase I (Pol I) to the rDNA promoter (Parlato et al., 2008). Genetic loss of TIF-IA impairs rRNA transcription, causing disruption of nucleoli resulting in severe growth deficits during embryonic development (Yuan et al., 2005). Several intracellular pathways converge on TIF-IA in response to either favorable or adverse conditions. As such TIF-IA activity is differentially regulated by various kinases including mTOR, ERK, AMPK, JNK (Zhao et al., 2003; Mayer et al., 2004, 2005; Hoppe et al., 2009) in response to environmental changes. Recently it has been shown that brain-derived neurotrophic factor (BDNF) regulates Pol I activity by ERK1/2 and TIF-IA activation stimulating hippocampal neuron morphogenesis (neurite length and branching) (Gomes et al., 2011).

Here, we have analyzed behavioral, cellular, and molecular changes in TIF-IA CaMKCreERT2 mutants characterized by the inducible ablation of TIF-IA gene in adult hippocampal neurons. Nucleolar stress, as a consequence of the inhibition of rRNA synthesis, results in hippocampal neurodegeneration (Parlato et al., 2008). However, the mutant mice do not show deficits in learning and memory behavioral tests. On the contrary, mutant mice present enhanced spatial learning, suggesting compensatory mechanisms. Moreover, hippocampal neurodegeneration is restricted and nucleolar integrity is restored. Increased neurogenesis in the DG could partially account for the lack of behavioral and morphological deficits. The molecular analysis suggests the activation of neuroprotective strategies to inhibit apoptosis and enhance survival. The mTOR pathway appears to be upregulated in the TIF-IA CaMKCreERT2 mutants, supporting the role of nucleolar activity in neuronal homeostasis.

\section{MATERIALS AND METHODS \\ MICE}

Homozygous TIF-IA flox $/$ flox $_{\text {CaMKCreERT2 }}^{\text {(TIF- }}$ IA $^{\text {CaMKCreERT2 }}$ ) mutant mice were generated by crossing mice carrying the TIF-IA floxed allele (TIF-IA ${ }^{\text {flox/flox }}$ ) to the transgenic line CaMKCreERT2. TIF-IA ${ }^{+/ \text {flox; CaMKCreERT2 }}$ positive mice were again crossed with TIF-IA ${ }^{\text {flox/flox }}$ mice. The

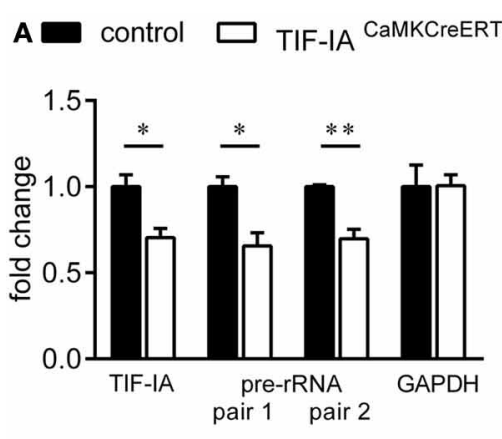

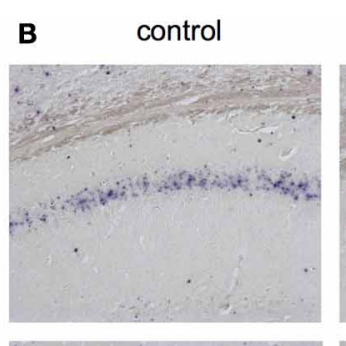

TIF-IA CaMKCreERT2
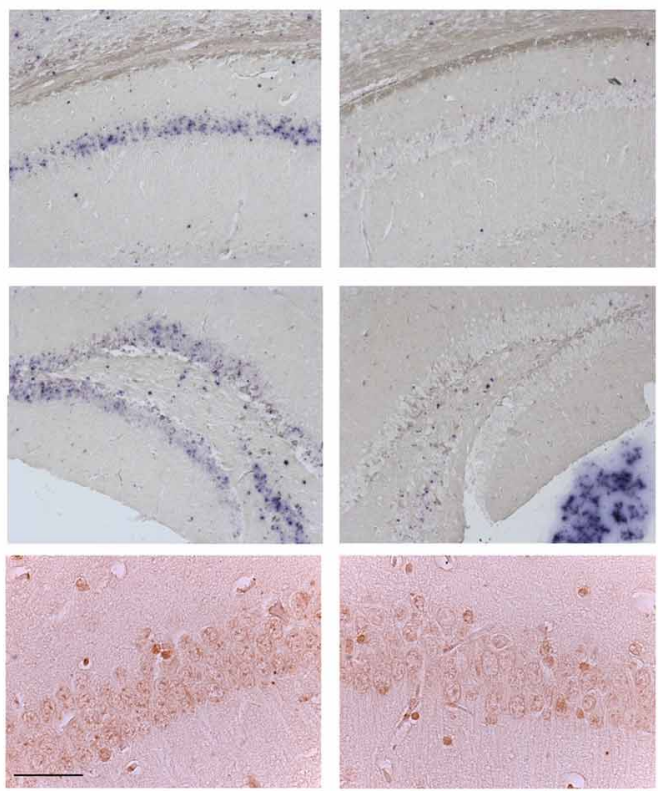

FIGURE 1 | Conditional ablation of TIF-IA reduces 47S pre-rRNA level in the hippocampus. (A) TIF-IA and GAPDH mRNA and pre-rRNA decrease by qPCR in the hippocampi of control and TIF-IACaMKCreERT2 mutants 1 month after tamoxifen. (B) Upper panels: Representative images showing the specific effects of TIF-IA loss on pre-rRNA synthesis (blue staining) in CA1 and DG by in situ hybridization in coronal paraffin sections of control and
TIF-IACaMKCreERT2 mutant mice 1 month after tamoxifen. Lower panels: TIF-IA protein analyzed by immunohistochemistry (IHC) in CA1 is visible in the nucleoli of controls but not in TIF-IA CaMKCreERT2 mutant mice. (C) $18 \mathrm{~S}$ rRNA analyzed by qPCR in control and TIF-IA CaMKCreERT2 mutants 1 month after tamoxifen is not affected; $n=5$. Error bars represent SEM. Scale bar: (B) upper panels: $150 \mu \mathrm{m}$; (B) lower panels: $60 \mu \mathrm{m} .{ }^{*} p<0.05 ;{ }^{* *} p<0.01$. 


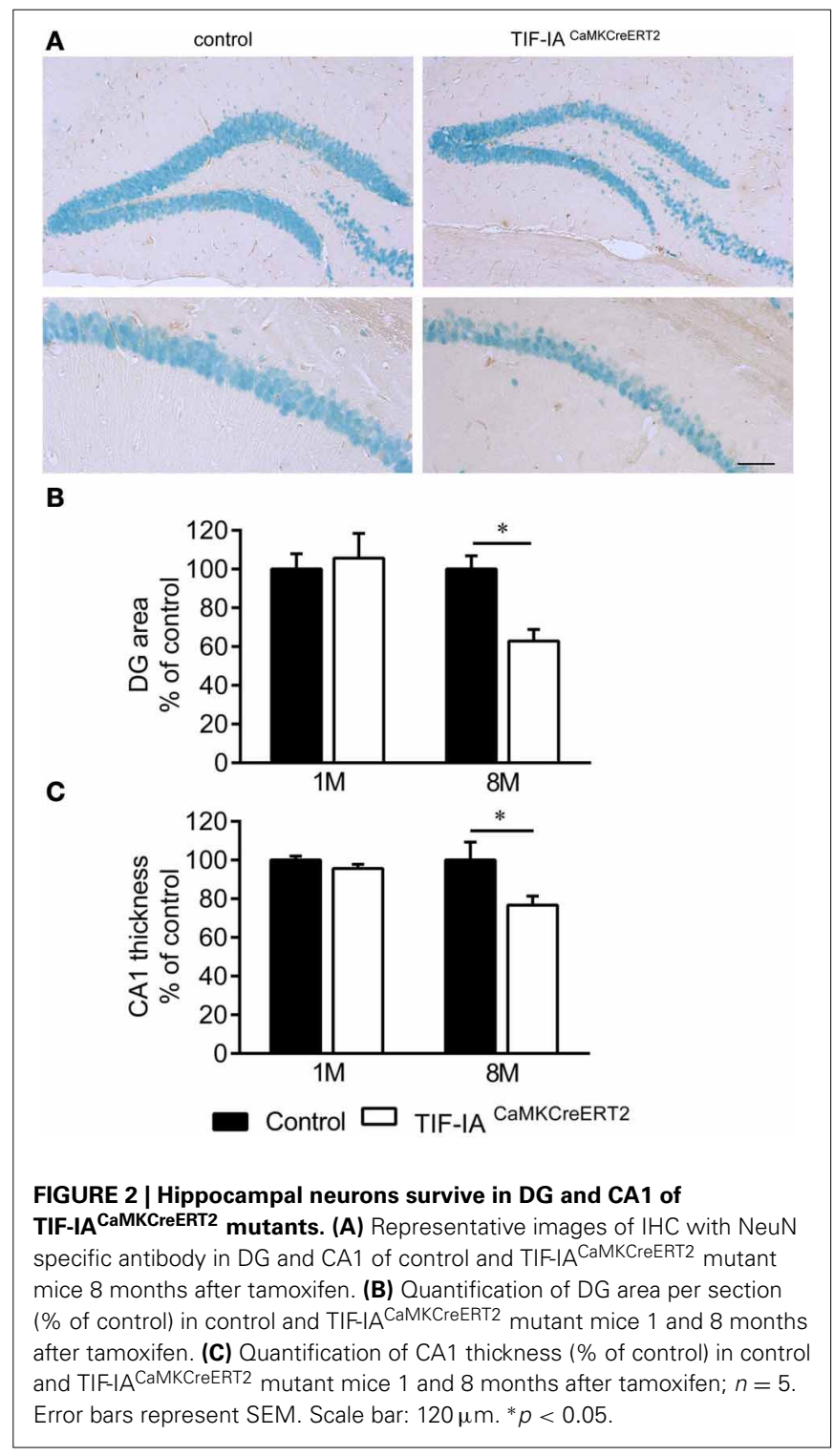

analysis of the genotype was performed as previously described (Parlato et al., 2008). 2-month-old TIF-IA ${ }^{\text {flox/flox }}$ control and TIF-IA CaMKCreERT2 mutant mice were both injected intraperitoneally with $1 \mathrm{mg}$ tamoxifen twice a day for five consecutive days and were analyzed at different time points after the last injection, in general by a $2-3$ month interval. Both male and female mice were used for the experiments. The mouse genetic background was $\mathrm{C} 57 \mathrm{Bl6} / \mathrm{N}$. The experiments were performed and analyzed blind with regard to the mouse genotype.

Procedures involving animal care were approved by the Committee on Animal Care and Use (Regierungspräsidium Karlsruhe) in accordance with the local Animal Welfare Act and the European Communities Council Directive of 24 November 1986 (86/609/EEC).

\section{BEHAVIORAL ANALYSIS}

The mice were tested at three time points: 5 weeks (controls, $n=16: 8$ females and 8 males; mutants, $n=15: 5$ females

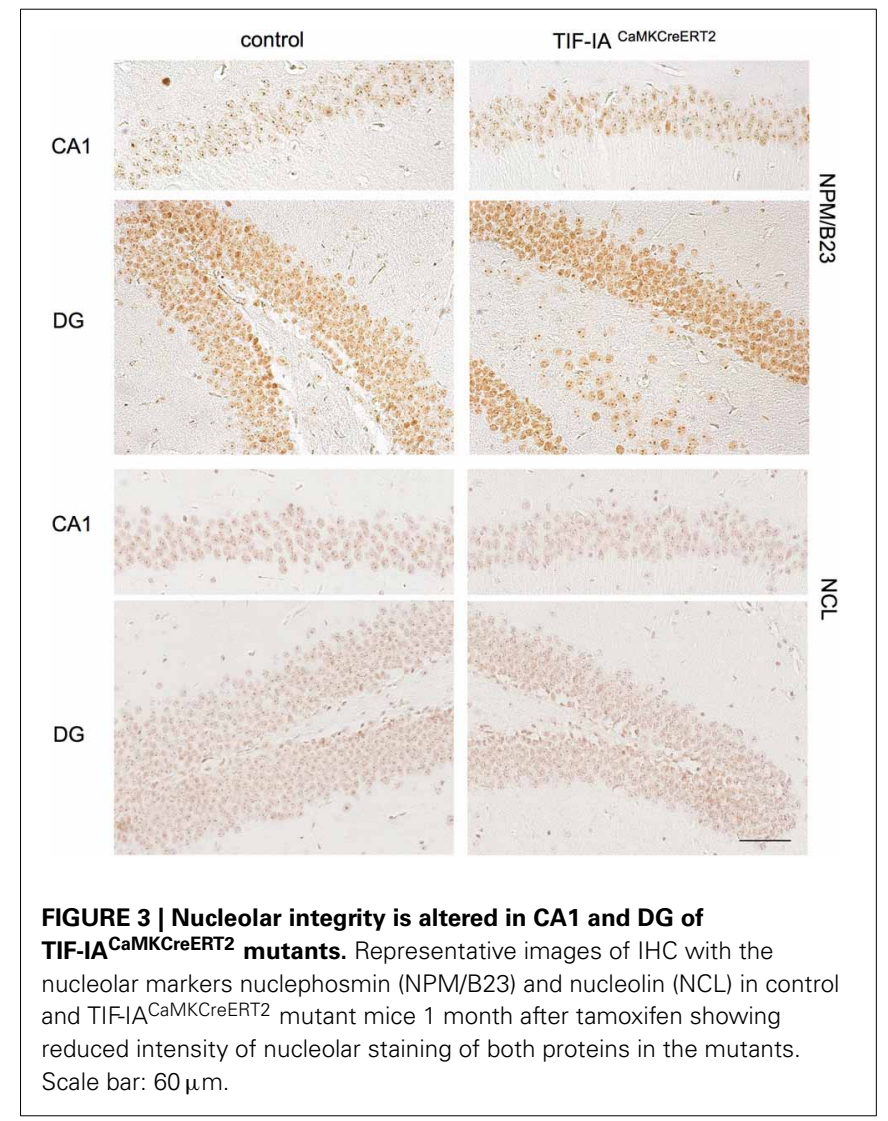

and 10 males), 3 months (controls, $n=6: 2$ females and 4 males; mutants, $n=5: 2$ females and 3 males), and 6 months (controls, $n=6: 4$ females and 2 males; mutants, $n=7: 4$ females and 3 males) after tamoxifen. Mice were subjected to behavioral examination in a battery of tests: handling, neurological assessment, Rotarod test, Open Field, Elevated Plus Maze, and Morris water maze test. In the last day of 1-week handling the mice were exposed to neurological assessment in order to find abnormalities in activity, reflexes and sense response, according to a table of neurological deficits (Katz et al., 1998).

For Rotarod test a standard apparatus (TSE systems, Bad Homburg, Germany) was used. Mice were habituated to the immobile rod (day 1) and then to the rod turning with slow speed $4 \mathrm{rpm}$ (day 2). In the 3rd day the test was performed and the rod was turning with increasing speed from 4 to $40 \mathrm{rpm}$. Time to fall from a rod was measured by the TSE system software.

For the Open Field, mice were placed in the center of gray round arena $(60 \mathrm{~cm}$ in diameter) surrounded by high walls $(30 \mathrm{~cm}$ high). The floor of the apparatus was virtually divided to 16 zones in EthoVision system (Noldus Information Technology, Wageningen, The Netherlands). The number of zones crossed by each mouse, the distance moved per minute of test, the total distance and time spent in center and periphery were also measured.

The level of anxiety was assessed in the Elevated Plus Maze test. The plastic apparatus consists of four arms $(30 \times 5.5 \mathrm{~cm}$ each $)$ 


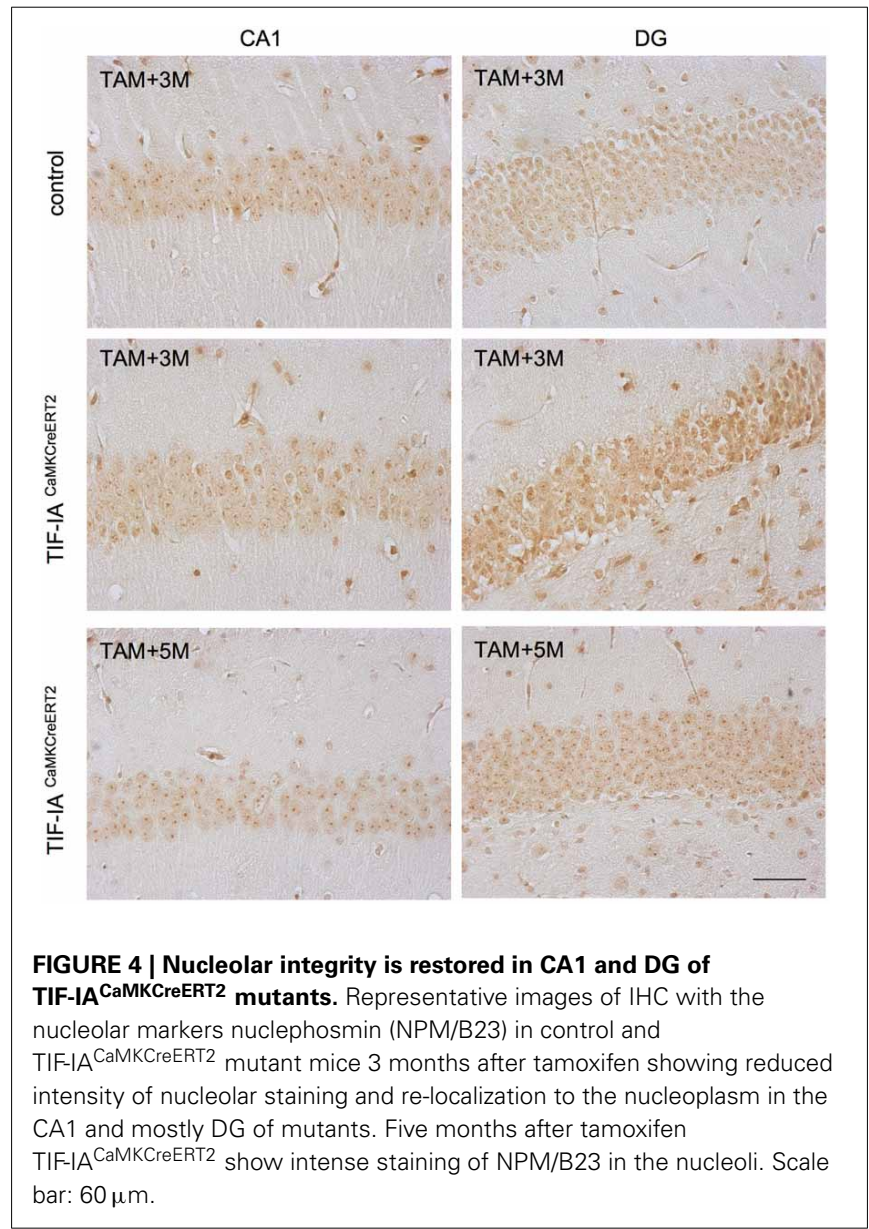

connected by central area $(5.5 \times 5.5 \mathrm{~cm})$. The mice were placed individually in the central area and were allowed to move freely for $5 \mathrm{~min}$. Time spent in each zone was measured by EthoVision system.

Morris water maze test was used for assessing hippocampusdependent memory. In the first day mice were allowed to swim in a pool with visible platform $(15 \mathrm{~cm}$ high, $11.5 \times 11.5 \mathrm{~cm})$ to assess visual and motoric abilities. The procedure was performed as previously described (Widy-Tyszkiewicz et al., 1993; Giese et al., 1998). Each mouse was exposed to 1 training session daily divided for 4 trials in a circular white pool (diameter $1.4 \mathrm{~m}$ ) with the platform submerged $1.5 \mathrm{~cm}$ under water $\left(24-27^{\circ} \mathrm{C}\right)$ in position 1 . Each mouse was trained to find a hidden platform in a latency of $60 \mathrm{~s}$. If mouse found a platform in $60 \mathrm{~s}$ or if the time elapsed, mouse was allowed to stay on the platform for $60 \mathrm{~s}$. Next, memory of platform position 1 was measured in $60 \mathrm{~s}$ probe trials 24 and $48 \mathrm{~h}$ after the 4th day of training. Mouse was put in the opposite quadrant to "platform quadrant" and was allowed freely swimming for $60 \mathrm{~s}$. After the second probe trail, 1-day training was performed to investigate the ability of re-learning to new platform location-position 2. The swim path and the time spent in four virtual quadrants of the pool were recorded by the EthoVision software.

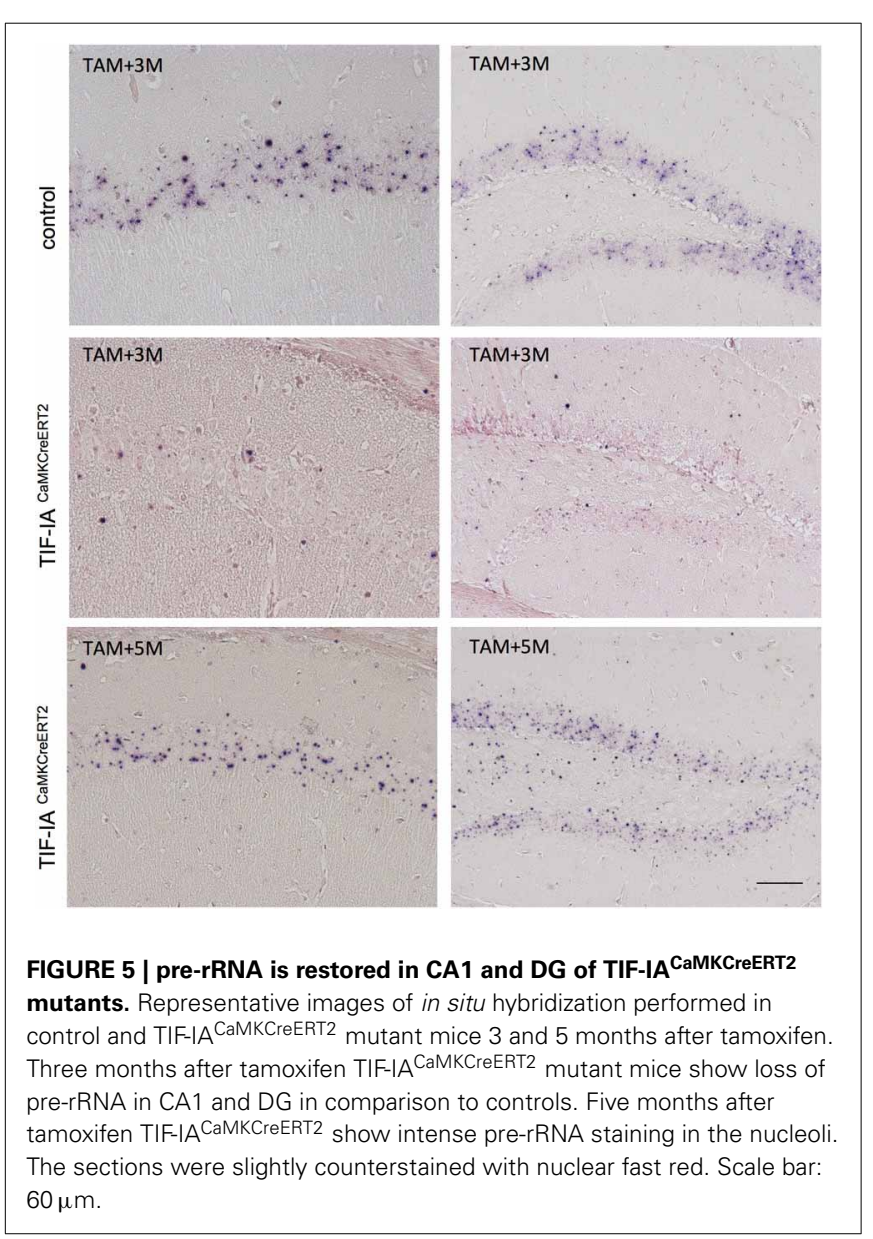

\section{ELECTROPHYSIOLOGY}

For in vitro long-term potentiation (LTP) recording animals 1 month after tamoxifen were anaesthetized with isoflurane and decapitated. The brains were quickly removed and placed in cold artificial cerebrospinal fluid (aCSF: $\mathrm{NaCl} 117 \mathrm{mM}$, $\mathrm{MgSO}_{4} 1.2 \mathrm{mM}, \mathrm{KCl} 4.7 \mathrm{mM}, \mathrm{CaCl}_{2} 2.5 \mathrm{mM}, \mathrm{NaHCO}_{3} 25 \mathrm{mM}$, $\mathrm{NaH}_{2} \mathrm{PO}_{4} 1.2 \mathrm{mM}, 10 \mathrm{mM}$ glucose, bubbled with carbogen). Both hemispheres were cut into $400 \mu \mathrm{m}$ coronal slices on a vibratome. Slices containing the hippocampus were placed in a recording interface chamber to recover for at least $1.5 \mathrm{~h}$ before recording. The slices were continuously perfused with carbogenated aCSF at $33^{\circ} \mathrm{C}$. Field excitatory postsynaptic potentials (fEPSPs) were recorded using glass micropipettes filled with $1 \mathrm{M} \mathrm{NaCl}$ yielding resistance of 2-3M 2 , in the stratum radiatum in CA1, evoked by stimulation of the Schaffer collateral-commissural afferents every $30 \mathrm{~s}$ with bipolar metal electrodes (test pulses at $0.033 \mathrm{~Hz}$, $0.1 \mathrm{~ms}$ ). Input-output (I-O) analysis was conducted to determine the maximal response to constant increase. During I-O protocol 16 stimuli with increasing intensity from 0 to maximum value were delivered. Intensity of the test stimulus was adjusted to obtain fEPSP with slopes that were one-third of the maximal response (established on the basis of I-O curve). Paired pulse facilitation experiments were performed before baseline recordings. PPF was examined at inter-stimulus intervals of 15, 25, 


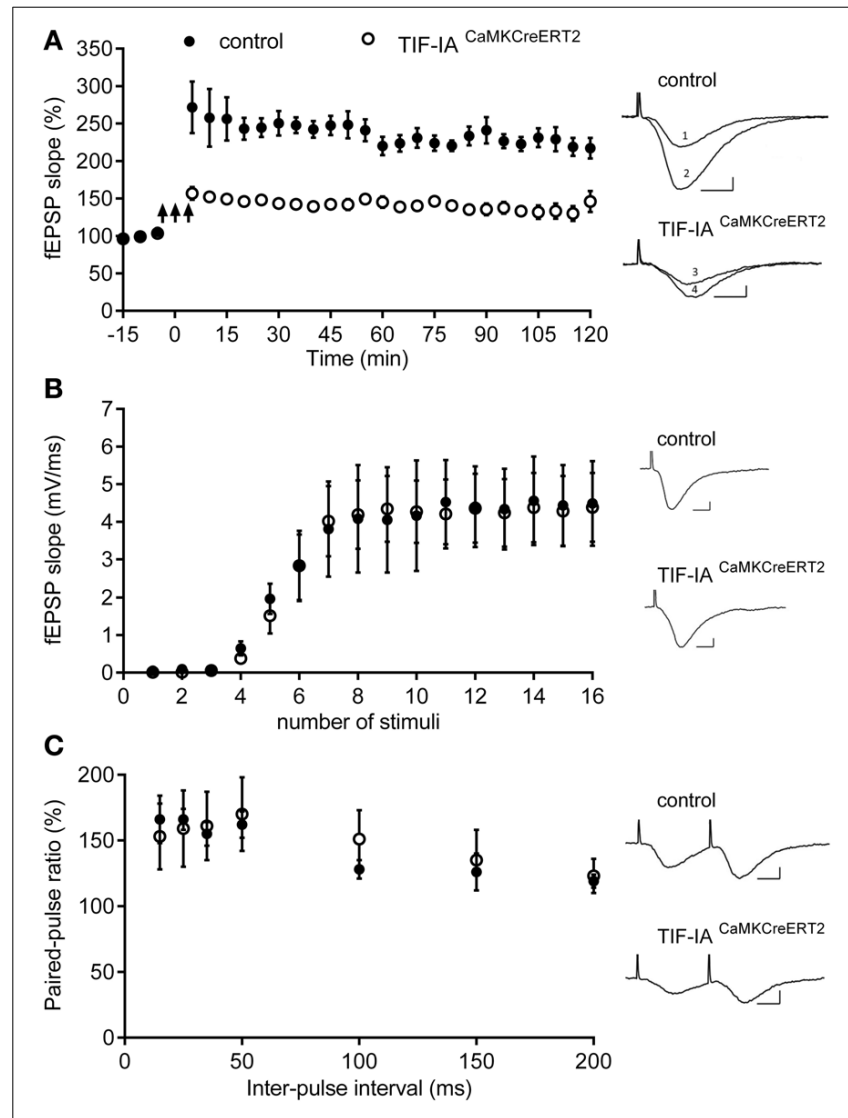

FIGURE 6 | TIF-IA CaMKCreERT2 mutant mice display hippocampal deficits in LTP. (A) Tetanically induced LTP is lower in TIF-IA CaMKCreERT2 mutant mice (open circles) in comparison with control group (filled circles) 1 month after tamoxifen. Arrows mark the stimulation $(3$ trains of $100 \mathrm{~Hz}$, $1 \mathrm{~s}$ stimulation separated by $3 \mathrm{~min} ; n=5$, control; $n=6$, mutant). Each point represents the average of 5 min recording from each animal group \pm SEM. Right panels: superimposed representative fEPSP traces recorded from control and TIF-IA CaMKCreERT2 mutant mice 10 min before LTP induction (1 and 3 , respectively) and 120 min after ( 2 and 4 , respectively). Scale bars: $5 \mathrm{~ms} / 2 \mathrm{mV}$. (B) Input-output (IO) curves representing the relationship between stimulus intensity and the slope of the fEPSP, did not differ significantly between control animals (filled circles; $n=5$ ) and TIF-IA CaMKCreERT2 mutant mice (open circles; $n=6$ ). Right panels: representative fEPSP traces with maximal slope, recorded from control (upper panel) and TIF-IACaMKCreERT2 mutant mice (bottom panel). Scale bars: $5 \mathrm{~ms} / 2 \mathrm{mV}$. (C) There were no differences in paired-pulse facilitation (PPF) ratios induced by delivering two stimuli with several different inter-stimulus intervals $(15,25,35,100,150$, and $200 \mathrm{~ms}$ ) between control animals (filled circles; $n=5$ ) and TIF-IA CaMKCreERT2 mutant mice (open circles; $n=6$ ). The PPF ratio represents the slope of the second fEPSP divided by the slope of the first fEPSP presented in \%. (Right panels) Representative fEPSP traces evoked by two stimuli with the same intensity and inter-stimulus interval $15 \mathrm{~ms}$, recorded from control (upper panel) and TIF-IACaMKCreERT2 mutant mice (bottom panel). Scale bars: $5 \mathrm{~ms} / 2 \mathrm{mV}$.

$35,50,100,150$, and $200 \mathrm{~ms}$ and stimulus intensity one-third of the maximal response. PPF (\%) was determined as a ratio of the second fEPSP slope to the first evoked. After at least $15 \mathrm{~min}$ of stable baseline LTP was induced tetanically (three trains of $100 \mathrm{~Hz} 1 \mathrm{~s}$ stimulation, separated by $3 \mathrm{~min}$ ). After the end of the tetanic protocol, a test pulse was subsequently applied for at least
$120 \mathrm{~min}$. Recordings were amplified and digitized and amplitudes of fEPSP were analyzed on-line and off-line. For analysis of LTP, the response slopes were expressed as a percentage of the average response slopes during the baseline period prior to LTP induction.

\section{GENE EXPRESSION PROFILING}

Array analysis of gene expression was performed on adult TIFIA CaMKCreERT2 mutant mice and control littermates $(n=5-6$ per each group) four weeks after injection of tamoxifen. Mice were killed by $\mathrm{CO}_{2}$, the brains were immediately removed and preserved in RNA-later solution (Ambion). Total RNA was isolated from hippocampal vibratome sections $(300 \mu \mathrm{m})$ using the RNeasy Mini Kit (Qiagen). Profiling was performed using the MouseWG-6 v2 BeadChip arrays (Illumina Inc., San Diego, CA, USA) according to the manufacturer's instructions. Microarray quality control was performed using the BeadArray R package from the Bioconductor suite (Gentleman et al., 2004). After background subtraction, the data were normalized using quantile normalization and then $\log 2$-transformed. Statistical analysis of differences in transcription was performed with limma from the Bioconductor suite. Ontology analyses were carried out using gene set enrichment analysis (GSEA) and identification of enriched putative transcription factor binding sites was performed using cREMaG. The data were stored in the GEO database (http://www.ncbi.nlm.nih.gov/geo/), record number GSE51240.

\section{HISTOLOGICAL ANALYSIS}

Mice were sacrificed by $\mathrm{CO}_{2}$ and brains were immediately dissected. For immunohistochemistry, brains were fixed in $4 \%$ paraformaldehyde overnight and paraffin embedded or sectioned on a vibratome (50 $\mu \mathrm{m}$ thickness), with the exception of paraffin sections that were $7 \mu \mathrm{m}$ thick. Sections from the region of the hippocampus comprised between Bregma $-1.46 \mathrm{~mm}$ and $-2.70 \mathrm{~mm}$ were incubated with primary antibodies overnight at $4^{\circ} \mathrm{C}$. Visualization of antigen-bound primary antibodies was carried out using either a biotinylated secondary antibody together with the avidin-biotin system and the VECTOR peroxidase kit (Vector Laboratories). Mice were intraperitoneally injected with 5-bromo-2-deoxyuridine (BrdU; Sigma) $40 \mathrm{mg} / \mathrm{kg}$ body weight in $0.1 \mathrm{M}$ Tris and then killed $2 \mathrm{~h}$ after a single BrdU injection $(n=3-4)$, or 2 weeks $(n=4)$, and 6 weeks $(n=4)$ after the last of 5 days of one daily BrdU injection. Primary antibodies for immunostaining were: anti-doublecortin (Santa Cruz), antibromodeoxyuridine (Dako), anti-Ki67, anti-nucleolin (Abcam), anti-nucleophosmin (NPM/B23), anti-neuronal-specific nuclear protein NeuN (Millipore), anti-phosphoS6 (S235/236) (Cell Signaling), anti-TIF-IA (Abnova). Non-radioactive in situ hybridization was performed on paraffin sections as previously described using a specific riboprobe hybridizing to regions in the leader sequence of the pre-rRNA (Parlato et al., 2008).

\section{RT-qPCR}

Total RNA was isolated from dissected hippocampi as for the gene expression profiling. Levels of TIF-IA mRNA and pre-rRNA were monitored by reverse transcription (RT) followed by quantitative PCR (qPCR). Synthesis of cDNA with M-MLV Reverse Transcriptase (Life Technologies) was 


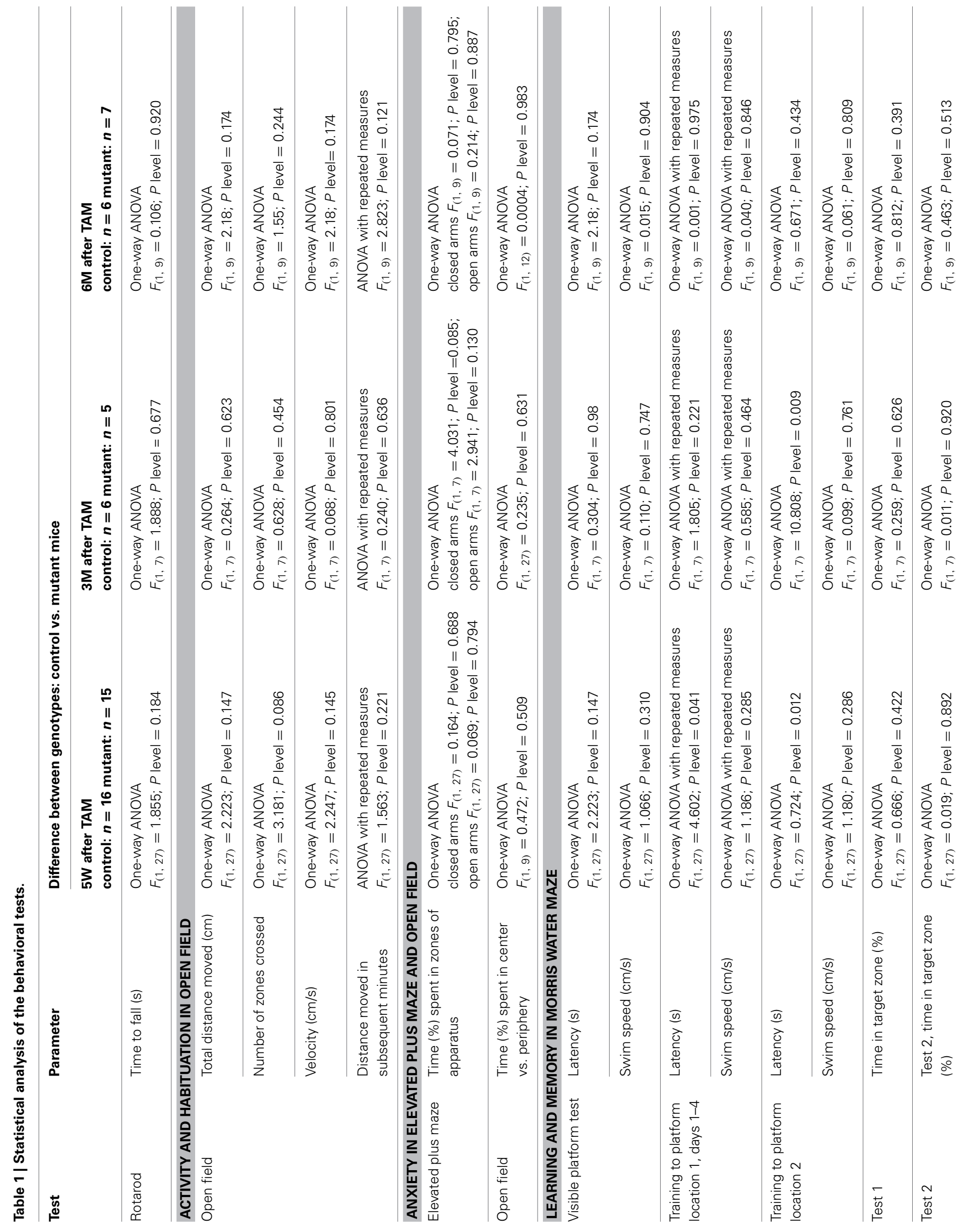




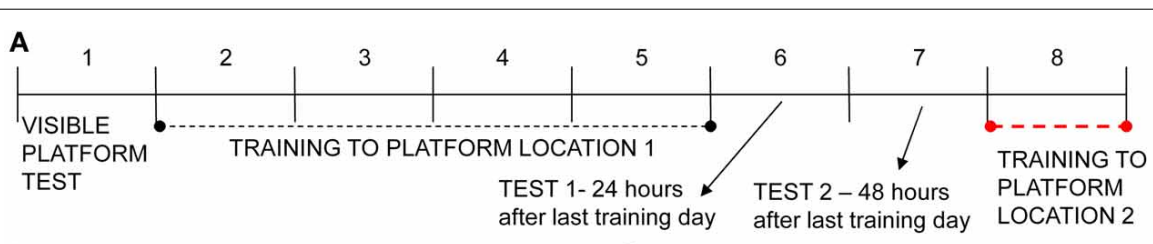

B

$\mathrm{TAM}+5 \mathrm{~W}$

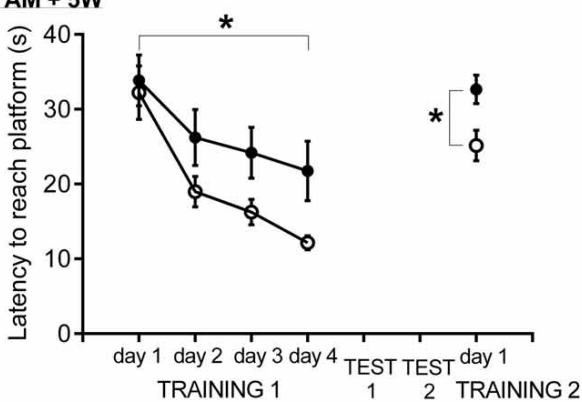

$\mathrm{TAM}+3 \mathrm{M}$

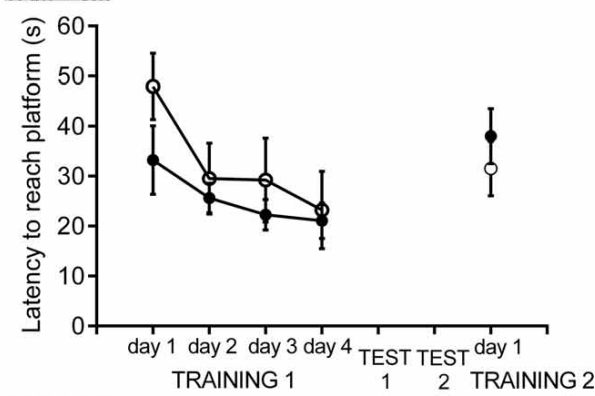

$\mathrm{TAM}+6 \mathrm{M}$

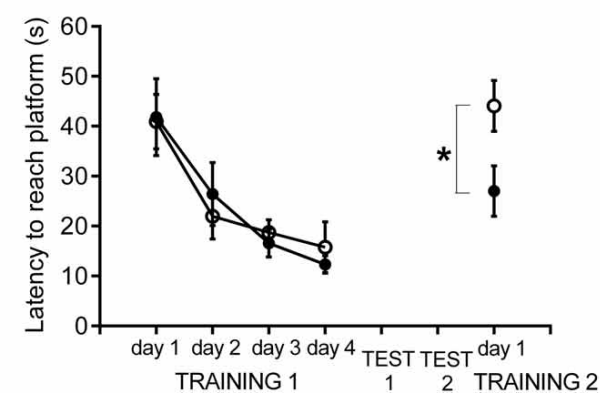

FIGURE 7 | TIF-IA CaMKCreERT2 mutant mice show enhanced learning. (A) Morris water maze procedure. Trials order in subsequent days. (B) Spatial learning in Morris water maze 5 weeks, 3 and 6 months after tamoxifen. Time to find a hidden platform in 4-day learning and 1-day re-learning is shown. Enhanced learning abilities in mutant compared to control mice $(p<0.05)$ were observed 5 weeks after induction of mutation. Deterioration in 1-day re-learning task was found in mutants 6 months after tamoxifen. No
C
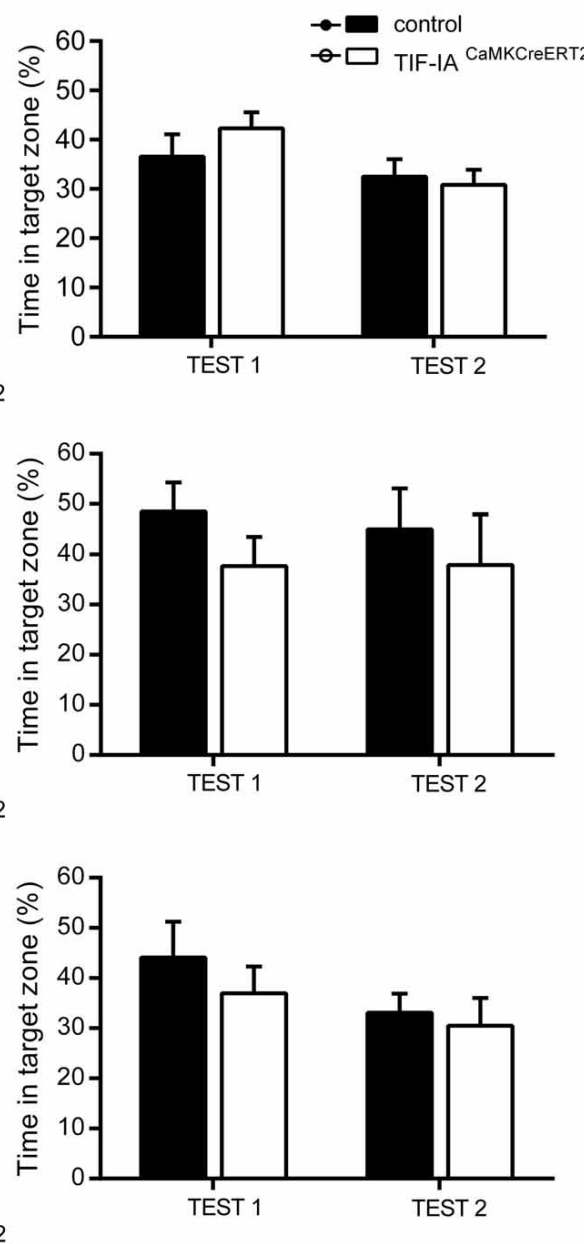

primed with random hexamers and $\mathrm{qPCR}$ was performed on a Light Cycler 480 instrument (Roche) using SYBR Green. TIF-IA mRNA primers (5'-GAACCCACTGAAGA TCTGCCTGC and 5'-GGAGTCTCCACCAGCGGTGCTG) were located in exons 14 and 15, respectively. The amplicon is disrupted upon Cre-mediated recombination due to deletion of exon 14. For detection of pre-rRNA, the first 130 nucleotides relative to the transcription start site were amplified using the $5^{\prime}$-ACTGACACGCTGTCCTTTCC and 5'-GACAGCTTCAGGCACCGCGA primers; a primer pair differences in swim speed were found. (C) Spatial memory tests in Morris water maze 5 weeks, 3 and 6 months after tamoxifen. Relative time of swimming in target quadrant (\%) is shown. Memory tests performed $24 \mathrm{~h}$ (Test 1 ) as well as $48 \mathrm{~h}$ (Test 2) after the last training day, showed memory for platform location in control and mutant mice tested in each time points after induction of mutation. Randomness level $(25 \%)$ is indicated by dotted line. $* p<0.05$. 
Table 2 | Analysis of gender differences in control and TIF-IA CaMKCreERT2 5 weeks after tamoxifen treatment.

\begin{tabular}{|c|c|c|c|}
\hline \multirow{2}{*}{$\begin{array}{l}\text { Test } \\
\\
\text { Visible platform test }\end{array}$} & \multirow{2}{*}{$\begin{array}{l}\text { Parameter } \\
\text { Latency (s) }\end{array}$} & \multicolumn{2}{|c|}{$\begin{array}{l}5 W \text { after TAM Controls, } \\
n=16: 8 \text { females, } 8 \text { males } \\
\text { Mutants: } n=15: 5 \text { females, } \\
10 \text { males }\end{array}$} \\
\hline & & Average & SEM \\
\hline & Control females & 14.09 & 3.53 \\
\hline & Control males & 13.5 & 5 \\
\hline & Mutant females & 12.81 & 6.49 \\
\hline & Mutant males & 12.37 & 9.64 \\
\hline & $\begin{array}{l}\text { Swim speed } \\
(\mathrm{cm} / \mathrm{s})\end{array}$ & Average & SEM \\
\hline & Control females & 19.9 & 1.09 \\
\hline & Control males & 20.45 & 0.56 \\
\hline & Mutant females & 20.88 & 0.95 \\
\hline & Mutant males & 18.54 & 0.59 \\
\hline \multirow{10}{*}{$\begin{array}{l}\text { Training to platform } \\
\text { location } 1 \text {, day } 4\end{array}$} & Latency (s) & Average & SEM \\
\hline & Control females & 27.79 & 7.33 \\
\hline & Control males & 15.74 & 2.06 \\
\hline & Mutant females & 13.03 & 1.48 \\
\hline & Mutant males & 11.72 & 1.21 \\
\hline & $\begin{array}{l}\text { Swim speed } \\
(\mathrm{cm} / \mathrm{s})\end{array}$ & Average & SEM \\
\hline & Control females & 20.71 & 1.09 \\
\hline & Control males & 21.25 & 0.56 \\
\hline & Mutant females & 21.68 & 0.95 \\
\hline & Mutant males & 19.34 & 0.59 \\
\hline \multirow{10}{*}{$\begin{array}{l}\text { Training to platform } \\
\text { location } 2\end{array}$} & Latency (s) & Average & SEM \\
\hline & Control females & 29.3 & 1.08 \\
\hline & Control males & 33.99 & 3.076 \\
\hline & Mutant females & 22.57 & 3.60 \\
\hline & Mutant males & 27.71 & 2.49 \\
\hline & $\begin{array}{l}\text { Swim speed } \\
(\mathrm{cm} / \mathrm{s})\end{array}$ & Average & SEM \\
\hline & Control females & 20.30 & 0.88 \\
\hline & Control males & 21.64 & 0.83 \\
\hline & Mutant females & 21.66 & 1.15 \\
\hline & Mutant males & 19.18 & 0.67 \\
\hline \multirow[t]{5}{*}{ Test 1} & $\begin{array}{l}\text { Time in target } \\
\text { zone (\%) }\end{array}$ & Average & SEM \\
\hline & Control females & 28.73 & 6.15 \\
\hline & Control males & 44.38 & 5.76 \\
\hline & Mutant females & 36.83 & 7.55 \\
\hline & Mutant males & 45.03 & 3.19 \\
\hline \multirow[t]{5}{*}{ Test 2} & $\begin{array}{l}\text { Time in target } \\
\text { zone }(\%)\end{array}$ & Average & SEM \\
\hline & Control females & 32.81 & 6.13 \\
\hline & Control males & 32.15 & 4.07 \\
\hline & Mutant females & 30.99 & 6.40 \\
\hline & Mutant males & 30.73 & 3.66 \\
\hline
\end{tabular}

changes were calculated as a fold change vs. mean of control samples.

\section{STATISTICAL ANALYSIS}

Behavioral and electrophysiological results were analyzed in Statistica 6.0 (StatSoft). One-Way ANOVA was used for Rotarod, Open Field, Elevated plus maze test and Two-Way ANOVA (genotype and gender) for relearning task in Morris water maze. For multiple comparisons in Morris water maze, the results were at first normalized to equalize variances in statistical program JMP v.5.0.1.2 followed by Two-Way ANOVA with repeated measures. Significant genotype effect was further analyzed using post-hoc NIR test. For electrophysiological recordings ANOVA with repeated measures was used. $P<0.05$ was acknowledged as a significance threshold. Data were pooled across animals of the same genotype and presented as mean \pm SEM using GraphPad Prism (GraphPad Software).

The ImageJ program was used to measure DG area and CA1 thickness, namely the average height of the CA1 layer. The DG area per section was measured on average in six coronal paraffin sections (one every 4 th sections) per mouse $(n=5)$. CA1 thickness was measured on average in six coronal sections (one every 4 th sections) per mouse $(n=5)$. In each section the CA1 thickness was measured in three different regions between the distal and proximal CA1. The number of phosho-S6 positive neurons in DG was measured as the number of double positive phospho-S6 and NeuN cells per section. In CA1 the number of phosho-S6 positive neurons in each section was counted between the distal and proximal CA1 in three area units used as a reference. The number of Ki67, BrdU, doublecortin positive cells in DG was measured as the average number of positive cells per section in five-six coronal vibratome sections (one every 3rd sections) per mouse $(n=4-5)$. For BrdU colocalization, the cells showing a nuclear BrdU staining (green) and surrounded by the doublecortin (brown) staining were counted in the DG. The results of the $t$-test are reported as follows: $\left({ }^{*} p<0.05{ }^{* *} p<0.01{ }^{* * *} p<0.001\right)$.

\section{RESULTS IMPAIRED rRNA SYNTHESIS RESULTS IN ABERRANT SYNAPTIC PLASTICITY}

Inducible conditional ablation of the transcription factor TIF-IA by the Cre-loxP system impairs rRNA transcription and nucleolar integrity in the adult hippocampus (Parlato et al., 2008). TIF-IA mRNA and pre-rRNA level analyzed by qPCR were significantly reduced in the TIF-IA CaMKCreERT2 mutant mice 1 month after induction of the mutation by tamoxifen injection. GAPDH mRNA used as a control does not change (Figure 1A).rRNA transcription monitored by in situ hybridization detecting the nascent $47 \mathrm{~S}$ pre-rRNA in hippocampal sections was strongly inhibited in the CA1 and DG 1 month after tamoxifen was given confirming our previous data (Parlato et al., 2008) (Figure 1B). Loss of TIF-IA nucleolar staining is evident in the mutants at the same stage; nonetheless without significantly altering mature $18 \mathrm{~S}$ rRNA level $(p=0.58)$ (Figures 1B,C). Increased level of the tumor suppressor $\mathrm{p} 53$ is associated with death of hippocampal neurons 3 months after tamoxifen treatment (Parlato et al., 2008) suggesting its role in neuronal survival. Although the DG and the CA1 are 


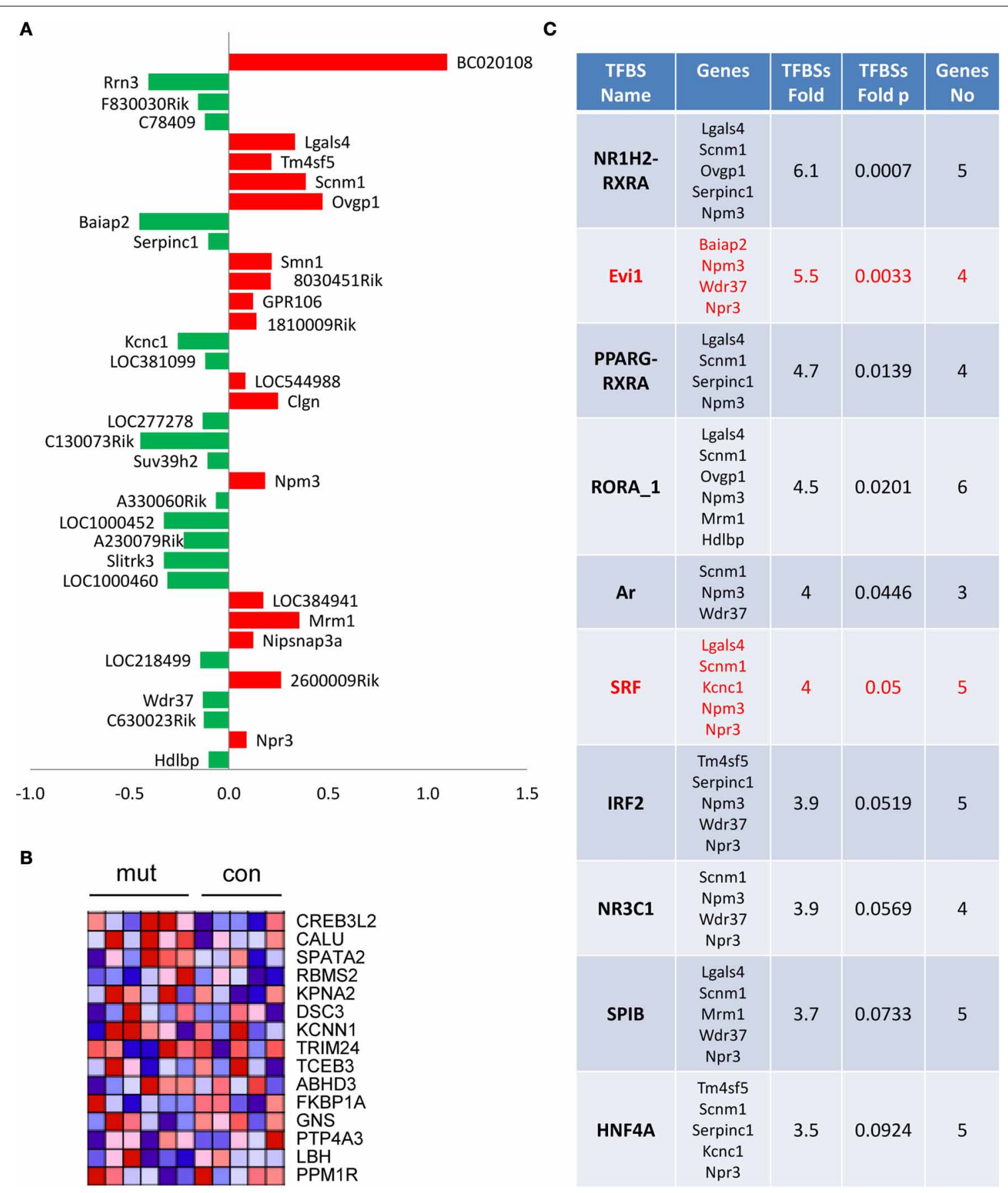

FIGURE 8 | Moderate effects on gene expression in TIF-IA CaMKCreERT2 mutant mice. One month after tamoxifen treatment. (A) Identification of 36 transcripts at unadjusted $p<0.001$ and their fold change vs. control group; (B) Heat map visualization of gene set enrichment analysis (GSEA) reveals changes in genes associated with vincristine resistance; (C) Identification of enriched putative transcription factor binding sites performed using cREMaG database. significantly smaller than controls in TIF-IA CaMKCreERT2 mutant mice 8 months after tamoxifen treatment, hippocampal neurons do not undergo massive degeneration as for example dopaminergic neurons lacking TIF-IA (Figures 2A,B) (Rieker et al., 2011). In agreement with this, p53 immunoreactivity does not increase at later stages (data not shown). This initial analysis suggested that hippocampal neurons were more resistant to nucleolar stress.

To further explore these observations we investigated the distribution of the nucleolar protein nucleophosmin NPM/B23, commonly used as a marker of nucleolar stress upon various 


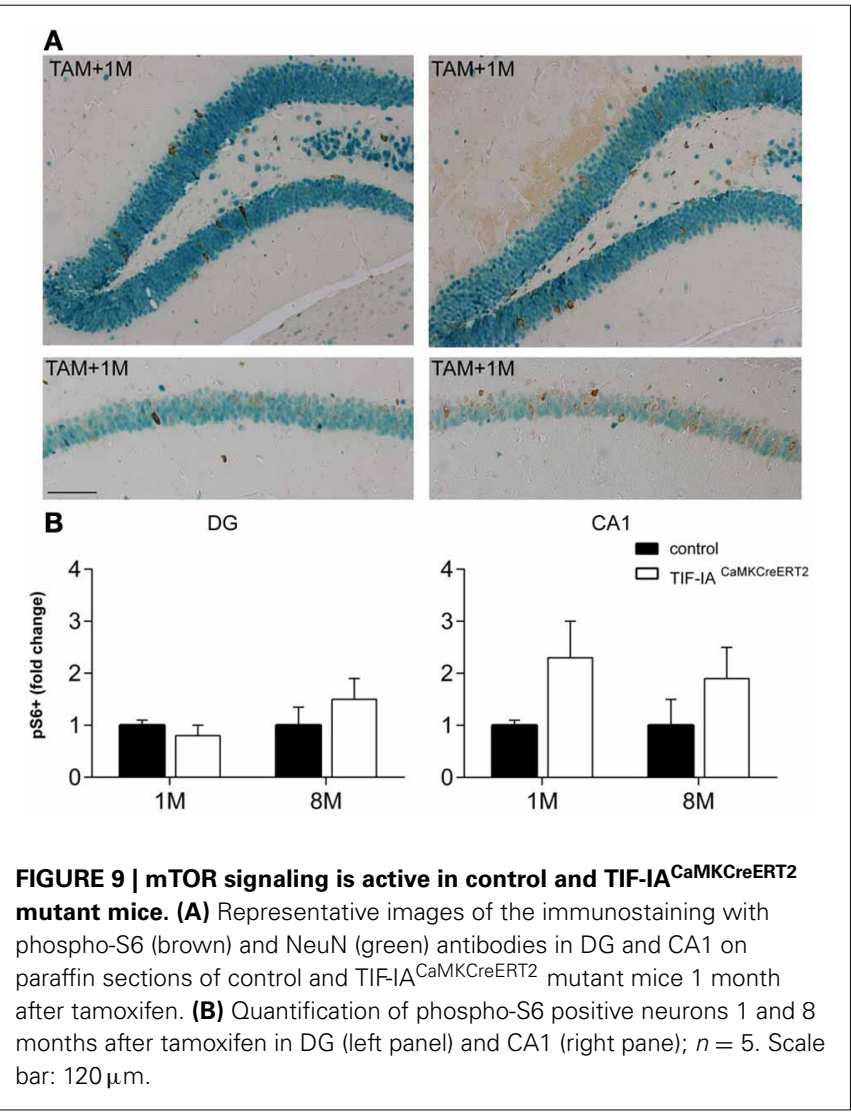

treatment and in different cellular contexts (Rubbi and Milner, 2003; Avitabile et al., 2011). Immunohistochemistry with NPM/B23 antibody shows that the controls are mainly characterized by an intense NPM/B23 staining in the nucleoli. In the DG increased NPM/B23 signal in the nucleoplasm is detectable in the subgranular zone (SGZ), occupied by sparse mitotic cells and it could be explained by loss of nucleolar integrity in dividing cells. One month after tamoxifen administration TIF-IA CaMKCreERT2 mutants showed reduced staining intensity in the nucleolus and increased signal in the nucleoplasm in comparison to controls (Figure 3). Notably, the nucleoplasmic distribution of NPM/B23 was more pronounced in the DG of the TIF-IA CaMKCreERT2 mutants (Figure 3).

To test whether other nucleolar proteins are affected at this stage, we analyzed the protein nucleolin (NCL), located in the granular component of the nucleolus and involved in various steps of ribosomal biogenesis (Abdelmohsen and Gorospe, 2012). NCL staining has been shown to decrease upon inhibition of rRNA biogenesis (Moore et al., 2013). Accordingly NCL intensity decreased in TIF-IA CaMKCreERT2 mutants supporting an initial induction of nucleolar stress 1 month after tamoxifen (Figure 3).

We have previously shown that NPM/B23 re-localizes in the nucleoplasm of TIF-IA CaMKCreERT2 mutants 3 months after tamoxifen concomitant with increased neuronal death (Parlato et al., 2008). To monitor the progression of this phenomenon, we compared controls and TIF-IA CaMKCreERT2 mutants 2.5 and 5 months after tamoxifen (Figure 4). As previously shown, a consistent loss of nucleolar NPM was visible in CA1 and DG of TIF-IA CaMKCreERT2 mutants 2.5 months after tamoxifen. However, NPM nucleolar staining in both regions was similar in controls and TIF-IA CaMKCreERT2 mutants 5 months after tamoxifen (Figure 4), suggesting that nucleolar stress could be rescued at later stages in TIF-IA CaMKCreERT2 mutants.

To further support this hypothesis we monitored rRNA transcription by in situ hybridization detecting $47 \mathrm{~S}$ pre-rRNA in hippocampal sections 2.5 and 5 months after tamoxifen (Figure 5). This approach confirmed a loss of pre-rRNA at 2.5 months in the mutants, while at 5 months we found pre-rRNA positive cells in both CA1 and DG (Figure 5). These results indicated that prerRNA and nucleolar stress could be rescued over time in this model, possibly halting neurodegeneration.

To determine whether inhibition of rRNA synthesis observed 1 month after tamoxifen affects synaptic plasticity, we applied a tetanic stimulation protocol to Schaffer collaterals in controls and TIF-IA CaMKCreERT2 mutant mice at this early stage. Tetanic stimulation potentiated fEPSPs in controls and TIF-IA CaMKCreERT2 mutant mice were stable throughout the experiment and lasted at least $120 \mathrm{~min}$. However, LTP in the mutant group was consistently lower ( $141 \pm 7 \% ; n=6)$ in comparison with the control group $(237 \pm 15 \% ; n=5)(p=0.001)$ (Figure 6). We also investigated the basic properties of synaptic transmission in both groups. There were no significant differences in the basic synaptic transmission (I-O curves) of fEPSP (Figure 6) between control and mutant mice. In addition we analyzed paired-pulse facilitation, a form of short-term plasticity. We found no differences in pairedpulse ratio between controls and TIF-IA CaMKCreERT2 mutants in all used inter-stimulus intervals $(15,25,35,50,100,150$, and $200 \mathrm{~ms})$.

\section{SENSORIMOTOR RESPONSES, ACTIVITY AND ANXIETY ARE UNAFFECTED IN TIF-IA CAMKCREERT2 MUTANT MICE}

To investigate the time-dependent consequences of nucleolar stress on hippocampal function, we analyzed behavioral, cellular, and molecular changes at different stages after induction of TIF-IA ablation.

Neurological examination starting 1 month after tamoxifen with an interval of 2-3 months revealed no difference between genotypes in home cage activity nor in functioning of all senses: olfactory, vision, hearing, and whisker movement. Motor coordination and balance were measured by the Rotarod test. TIF-IA ${ }^{\text {CaMKCreERT2 }}$ mutant mice retained normal motoric functions and coordination as they showed similar endurance to controls (Table 1). In new, open space (Open Field test) TIFIA CaMKCreERT2 mutant mice were as active as controls. During 5-min test controls and mutants covered similar distance and number of zones (Table 1). Also habituation to a new environment measured as a distance traveled in each minute of test was not changed in the mutant mice (Table 1). Anxiety to open, elevated space was assessed by 5-min exposure to Elevated Plus Maze. Mutant and control mice spent both more time in the closed arms than in the open arms of the apparatus showing similar level of anxiety (Table 1). This observation was confirmed by assessing anxiety level in the open field as time spent in the central vs. the periphery zone. Independently of the mutation, the 


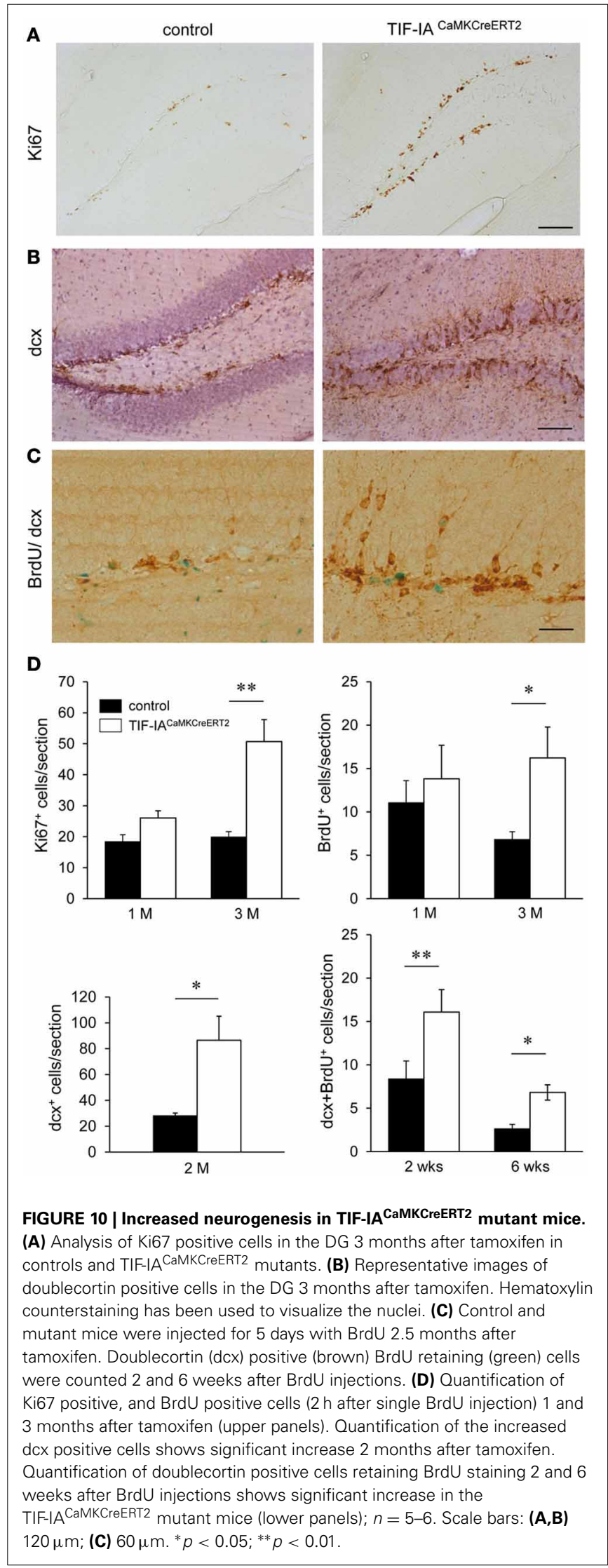

mice spent less than $10 \%$ of time in the aversive zone of open field showing strong preference for the periphery zone (Table 1).

\section{LOSS OF TIF-IA ENHANCES SPATIAL LEARNING AND RE-LEARNING IN MORRIS WATER MAZE}

In the visible platform test, all mice showed no motivational, visual, or motoric problems in reaching the platform. Both TIFIA ${ }^{\text {CaMKCreERT2 }}$ mutant and control mice found the visible platform in less than $15 \mathrm{~s}$ (Table 1). Spatial learning was assessed during the training phase by latency to reach the platform (Figure 7A) and swim speed (Table 1). While swim speed was similar between genotypes, we found differences in time to find the hidden platform. Five weeks after induction of TIF-IA mutation, we observed a better performance of TIF-IA CaMKCreERT2 in comparison to controls and to other time points (Figure 7B, Table 1). In the first day of training both mutant and control mice required similar time to find the platform. However, from the 2nd to 4th day of training, the TIF-IA ${ }^{\text {CaMKCreERT2 }}$ mutant mice were able to reach the platform in less than $20 \mathrm{~s}$, while the controls needed longer time. Also in re-learning task, the mutants found the platform in a new location (position 2) faster than control mice (Figure 7B). However, mutants tested 3 and 6 months after induction of the mutation, improved their time to find a platform similarly to controls. No differences were found in 1day of reversal learning between TIF-IA ${ }^{\text {CaMKCreERT2 }}$ mutant mice and controls 9 weeks after induction of the mutation. However, 6 months after tamoxifen, TIF-IA ${ }^{\text {CaMKCreERT2 }}$ mutant mice showed worsened re-learning abilities in comparison to control mice (training 2, Figure 7A). In this case the mice experienced 1 day of re-training to learn a new platform position (position 2). TIFIA CaMKCreERT2 mutant mice spent significantly longer time to find the platform new location than control mice (Figure 7B, Table 1).

Spatial memory was evaluated in 2 probe trials (Figure 7A). The platform was removed and the time spent in different quadrants of the pool was measured. In the first probe trial ( $24 \mathrm{~h}$ after the last training day) both mutant and control mice showed memory of the platform position, as they selectively searched for a platform in the proper quadrant for longer than $25 \%$ (chance level) of total time swimming. $48 \mathrm{~h}$ after the last training day, in the second probe trial, all mice spent more than $25 \%$ of time in the proper quadrant (Figure 7C). No differences between genotypes were found in quadrant preferences at any time points after ablation of TIF-IA gene (Table 1).

Sexual dimorphism in spatial learning has been observed in several studies, in particular mouse males showed reduced latencies than females at the initial learning stages (Schmidt et al., 2009; Chow et al., 2013). To address this aspect in control and TIF-IA CaMKCreERT2 mutants we analyzed gender differences after tamoxifen treatment (Table 2). Despite some apparent trends in "Training to platform location 1," based on Two-Way ANOVA we could not detect any significant differences between males and females; for example over 1-4 training days we observed the following: Latency (s): "genotype" $F_{(1,27)}=4.602 ; p=0.041$, "sex" $F_{(1,27)}=$ 
2.426; $p=0.130$, "Interaction sex* genotype" $F_{(1,27)}=2.115$; $p=0.157$.

\section{HIPPOCAMPAL NEURONS ADOPT EARLY COMPENSATORY MECHANISMS IN RESPONSE TO NUCLEOLAR STRESS}

To further investigate the early molecular alterations caused by inhibition of rRNA transcription, we analyzed changes in gene expression 1 month after tamoxifen injection in control and TIF-IA ${ }^{\text {CaMKCreERT2 }}$ mutants (Figure 8). Gene expression profiling analysis showed moderate effects of the mutation on gene expression (36 transcripts differentially expressed at $p<0.001$, as compared to the control group). Interestingly, among these transcripts we found a significant decrease in the TIF-IA/Rrn3 mRNA. In line with normal neuronal survival at this stage we did not detect any genes related to neuronal death, inflammation or gliosis (Figure 8A). Nevertheless, GSEA revealed similarities between mutation-induced changes in gene expression and some of the previously reported expression patterns elicited by cellular stress (Figure 8B). Additionally, enrichment in putative serum response factor (SRF) binding sites in the promoters of the genes with changed expression was observed, indicating potential adaptation in the mitogen-activated protein kinase cascade which could be regarded as a pro-survival response (Figure $\mathbf{8 C}$ ).

\section{HIPPOCAMPAL NEURONS RESPOND TO NUCLEOLAR STRESS BY ACTIVATION OF THE mTOR SIGNALING}

Because we have recently reported that loss of TIF-IA leads to downregulation of mTOR activity and neurodegeneration of dopaminergic neurons (Rieker et al., 2011), we have analyzed mTOR activity in CA1 and DG of TIF-IA CaMKCreERT2 mutants and controls 1 and 8 months after tamoxifen to establish how this pathway responds to nucleolar stress in hippocampal neurons. Phosphorylation of the ribosomal S6 protein is a well-known marker of mTOR activation. Therefore, we have performed immunohistochemistry with NeuN and pS6 specific antibodies and counted the number of NeuN positive cells also labeled by phosphoS6 (Figure 9). In contrast to dopaminergic neurons lacking TIF-IA, mTOR activity was not down-regulated at any stages in hippocampal neurons, and it rather appeared up-regulated in TIF-IA CaMKCreERT2 mutants (Figure 9B). This analysis suggests that increased levels of pS6 and mTOR activity may be a compensatory response to nucleolar stress in hippocampal neurons.

\section{NEUROGENESIS INCREASES IN TIF-IA CAMKCREERT2 MUTANTS}

Adult neurogenesis in the SGZ of the hippocampus occurs in response to environmental changes and during disease progression (Lledo et al., 2006). As nucleolar stress in the TIFIA ${ }^{\text {CaMKCreERT2 }}$ mutants does not lead to complete loss of hippocampal neurons or of their function (Figures 2, 7), we analyzed neurogenesis in the hippocampus of controls and TIF-IA CaMKCreERT2 mutants at different stages after tamoxifen. By immunohistochemistry based on the proliferation marker Ki67 we detected increased proliferating cells in the DG of TIF-IA ${ }^{\text {CaMKCreERT2 }}$ mutants 2.5-3 months after tamoxifen (Figures 10A,D). In this set of mice DG area was not significantly different in the mutants 1 month after tamoxifen ( $89 \%$ of controls, $p=0.3$ ) and it was reduced 3 months after tamoxifen
( $80 \%$ of controls, $p=0.0006$ ). We showed increased proliferating cells also by normalization of the Ki67 labeled cells to the DG area of the respective section $\left(\mathrm{mm}^{2}\right)$ (control $1 \mathrm{M}$ : $88.5 \pm 5.3$; mutant $1 \mathrm{M}: 144 \pm 21, p=0.06$ and control $3 \mathrm{M}: 98.7 \pm 15$; mutant $3 \mathrm{M}: 311.9 \pm 44, p=0.01)$. This increase was confirmed by the higher number of bromodeoxyuridine (BrdU) positive cells

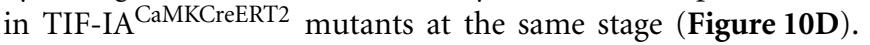
Increased neurogenesis was supported by the significantly higher number of doublecortin positive cells, a marker of immature neurons in TIF-IA ${ }^{\text {CaMKCreERT2 }}$ mutants (Figures 10B,D).

In adult mice the maturation of newly generated hippocampal neurons in the DG is complete by 4 months of age (van Praag et al., 2002; Laplagne et al., 2006); nevertheless neuronal properties emerge between 4 and 8 weeks. To establish whether increased BrdU labeling reflects increased neurogenesis we analyzed the number of doublecortin positive cells retaining BrdU staining 2 and 6 weeks after labeling. The increased number of double positive cells in the TIF-IA ${ }^{\text {CaMKCreERT2 }}$ mutants (Figures 10C,D) indicated higher generation of immature neurons. We showed similar results by normalization of the labeled cells to the DG area of the respective section $\left(\mathrm{mm}^{2}\right)$ ( control 2 weeks: $43.5 \pm 7.5$; mutant 2 weeks: $104 \pm 8, p=0.001$ and control 6 weeks: $13.7 \pm$ 2.3; mutant 6 weeks: $40.3 \pm 5.7, p=0.01$ ).

\section{DISCUSSION}

Inhibition of rRNA synthesis and nucleolar morphology is associated with aging and several neurological and psychiatric disorders linked to altered protein synthesis including neurodegenerative diseases, autism spectrum disorders (ASD), and depression (Hetman and Pietrzak, 2012; Parlato and Kreiner, 2013). In this study we showed that hippocampal neurons adopt a variety of cellular and molecular strategies to preserve their function in response to inhibition of rRNA transcription and nucleolar stress. These adaptive mechanisms include increased neurogenesis in the DG, early activation of neuroprotective pathways and late recovery of rRNA transcription.

The TIF-IA ${ }^{\text {CaMKCreERT2 }}$ mutants represent a unique model to investigate the effects of perturbed nucleolar activity on protein translation and synaptic transmission at different stages. We identified three major stages: an initial phase characterized by pre-rRNA transcription inhibition and incipient nucleolar stress, reduced LTP but no effects on neuronal survival and improved learning performance ( 1 month after tamoxifen), a second phase characterized by nucleolar stress linked to a peak of neurodegeneration and neurogenesis (3 months after tamoxifen), and a third phase in which nucleolar function and integrity is restored and neurodegeneration is halted (5-8 months after tamoxifen).

In particular, concomitant with the first phase we observed shorter escape latencies in TIF-IA CaMKCreERT2 mutants than controls during acquisition and reversal of Morris water maze. At the same stage the mutants also showed enhanced (re-)learning abilities in spatial tasks. However, in the probe tests TIF-IA CaMKCreERT2 mice performed as well as controls. Interestingly, improved learning performance was not correlated with enhanced synaptic plasticity, as LTP was decreased compared to the control group indicating that TIF-IA may play a role in the establishment of LTP. Although LTP is a cellular mechanism of 
the learning process and its enhancement in general accompanies learning, some exceptions have been reported in line with our results. A similar phenomenon to that observed in TIFIA CaMKCreERT2 mice was for example documented in the GluR1 knock-out mice. In this model LTP was absent at the synapses formed between CA3-CA1, whereas spatial learning tested in the Morris water maze was not affected (Zamanillo et al., 1999; Reisel et al., 2002; Sanderson et al., 2008). In TIF-IA CaMKCreERT2 model the molecular basis of this dissociation between LTP and improved learning requires further investigation.

Enhanced adult hippocampal neurogenesis could account for the learning and memory similarities between controls and mutant mice observed in the second phase. It has been previously shown that neurogenesis and learning of water maze task are correlated (Kempermann and Gage, 2002). New neurons in adult hippocampus may provide better flexibility for the acquisition of spatial platform position and for learning a changed platform position (reversal) (Garthe et al., 2009). This flexibility, however, was not sustained over time, as TIF-IA CaMKCreERT2 mice 6 months after tamoxifen show deterioration of reversal learning.

In addition the TIF-IA CaMKCreERT2 mutants allow to explore how newly generated neurons adapt to the local environment. In other mouse models of hippocampal neurodegeneration such as the conditional mutant mice lacking the pro-survival transcription factors CREB (cAMP-responsive element binding protein) and CREM (cAMP-responsive element modulator) in the postnatal forebrain (Mantamadiotis et al., 2002), hippocampal neurons undergo massive neurodegeneration, despite increased neurogenesis in the DG (Luzzati et al., 2011). In fact, the newly generated neurons are also affected by the constitutive loss of CREB upon expression of the Cre recombinase leading to their death. The TIF-IA CaMKCreERT2 mutants are inducible upon injection of tamoxifen; therefore the newly generated neurons do not carry the TIF-IA mutation and are provided with an intact RNA polymerase I transcription apparatus. Certainly it is puzzling how neurons can initially survive and function without pre-rRNA synthesis and how pre-rRNA synthesis is restored. Although further studies are required to establish whether increased neurogenesis accounts for keeping intact spatial learning and memory in TIFIA CaMKCreERT2 mutants and nucleolar function, other scenarios should be envisaged.

An intriguing hypothesis is that ribosomes are transported at the degenerating synapses by either other neurons or glial cells. This mechanism has been recently described between Schwann cells and regenerating axons, suggesting a potential compensatory mechanism in response to rRNA synthesis perturbation (Court et al., 2008, 2011).

Another explanation of the confined nucleolar effects on neuronal survival and function in the hippocampus could be that the DG is a region with low activity in which only a low proportion of neurons are active. On the contrary immature neurons in adult hippocampus show increased activity and high excitability as during development (Piatti et al., 2013). Thus, the combination of these two factors could account for hippocampal adaptation.

Concomitantly, loss of TIF-IA could be compensated by the induction of a transcription factor playing a similar role in the regulation of RNA Pol I activity and/or mechanisms altering the turn-over of ribosomes and pre-rRNA processing could participate to the maintenance of protein synthesis (Granneman and Baserga, 2005; Kopp et al., 2007). A possible candidate is the NPM/B23 itself; in response to inhibition of RNA polymerase I activity NPM/B23 has been reported to complex with the mRNAbinding proteins hnRNPU and hnRNPA1 in the cytoplasm influencing rRNA processing and sustaining cell survival (Yao et al., 2010). Moreover NPM/B23 may regulate rRNA transcription by its association with the rDNA promoter and function as a histone chaperone (Murano et al., 2008). Decreased level of NPM protein independently of mRNA differences has been reported as a consequence of kainic-acid induced excitoxicity in hippocampal CA1 and NPM/B23 overexpression is neuroprotective against this insult, suggesting an important function in survival of hippocampal neurons (Marquez-Lona et al., 2012). We cannot exclude that reduced NPM/B23 is accompanied by reduced protein expression in the nucleoli in TIF-IA CaMKCreERT2 mutants.

Interestingly, nucleolar hypertrophy is linked to neuronal resistance to neurotoxic $\beta$-amyloid deposits and $A \beta$ plaques in the CA1 of asymptomatic AD subjects; on the contrary in $\mathrm{AD}$ there is a significant atrophy of the nucleoli in the CA1 (Iacono et al., 2008). These examples indicate multiple mechanisms sustaining nucleolar activity in hippocampal neurons under stress that require further investigation.

At the intracellular level, the upregulation of the MAPK and mTOR pathways might limit the effects of disrupted rRNA transcription steering the response toward maintenance of functional protein synthesis and neuronal survival and activity (Mendoza et al., 2011). Because here we monitor S6 phosphorylation at Ser235/236 that are target sites common to RSK and S6K, we cannot exclude that upregulation of Ras-ERK pathway promotes the compensatory response, as also suggested by the gene expression profiling analysis. Upstream regulators of mTOR include growth factors, nutrients, energy and stress and a wired network involving PI3K/Akt and Ras/ERK to control translation, ribosome biogenesis, autophagy, transcription, and metabolism (Wullschleger et al., 2006). The molecular basis of enhanced mTOR and/or ERK activity in TIF-IA CaMKCreERT2 mice remains as of yet unknown. Nevertheless, enhanced mTOR activity is common to ASD (Kelleher and Bear, 2008). Interestingly, genetically increasing the levels of eukaryotic translation initiation factor $4 \mathrm{E}$ (eIF4E) in mice results in exaggerated cap-dependent translation and behaviors reminiscent of autism (Santini et al., 2013). Future research should address how nucleolar activity is regulated in the pathophysiology of ASD and other brain disorders in which global or local protein synthesis is upregulated.

Certainly, the mechanisms enabling neurons to survive despite inhibition of pre-rRNA synthesis will help to better understand neuronal stress response and defense strategies. Notably, although TIF-IA deficient dopaminergic and dopaminoceptive neurons undergo virtually complete loss, these neurons still survive several months without pre-rRNA synthesis, despite a strong nucleolar stress response triggering high level of p53 (Rieker et al., 2011; Kreiner et al., 2013). Nucleolar stress in dopaminoceptive neurons leads for example to autophagy mediated by p53-mediated mTOR inhibition, extending neuronal survival (Kreiner et al., 2013). 
In conclusion, our data reveal an intricate series of events potentially relevant for therapeutic applications in neurological and psychiatric disorders related to disturbances of the hippocampal translational machinery.

\section{ACKNOWLEDGMENTS}

We thank Dr. Evgeni Nikolaev for assistance with the statistical analysis, Dr. Kerry L. Tucker and Prof. Joachim Kirsch for insightful discussions and facilities. This work was supported by the German Federal Ministry for Education and Research (BMBF) through the NGFNplus grant FKZ 01GS08142, the DKFZ Intramural Funding Program to Rosanna Parlato, the statutory funds of the Department of Brain Biochemistry, Institute of Pharmacology, PAS, the Alfried Krupp Prize to Birgit Liss, the Polish National Science Center grant (SONATA) 2011/01/D/NZ4/03744 to Witold Konopka, the European Union structural funds: Innovative Economy Operational Program \#8212; Project No. POIG.01.01.02-00$109 / 09$ to Leszek Kaczmarek. The project based on this report was also funded by the program for medical genome research with financial support from BMBF under the support code 01GS08141. The author is responsible for the content of this publication.

\section{REFERENCES}

Abdelmohsen, K., and Gorospe, M. (2012). RNA-binding protein nucleolin in disease. RNA Biol. 9, 799-808. doi: 10.4161/rna.19718

Avitabile, D., Bailey, B., Cottage, C. T., Sundararaman, B., Joyo, A., McGregor, M., et al. (2011). Nucleolar stress is an early response to myocardial damage involving nucleolar proteins nucleostemin and nucleophosmin. Proc. Natl. Acad. Sci. U.S.A. 108, 6145-6150. doi: 10.1073/pnas. 1017935108

Cajigas, I. J., Will, T., and Schuman, E. M. (2010). Protein homeostasis and synaptic plasticity. EMBO J. 29, 2746-2752. doi: 10.1038/emboj.2010.173

Chow, C., Epp, J. R., Lieblich, S. E., Barha, C. K., and Galea, L. A. (2013). Sex differences in neurogenesis and activation of new neurons in response to spatial learning and memory. Psychoneuroendocrinology 38, 1236-1250. doi: 10.1016/j.psyneuen.2012.11.007

Court, F. A., Hendriks, W. T., MacGillavry, H. D., Alvarez, J., and van Minnen, J. (2008). Schwann cell to axon transfer of ribosomes: toward a novel understanding of the role of glia in the nervous system. J. Neurosci. 28, 11024-11029. doi: 10.1523/JNEUROSCI.2429-08.2008

Court, F. A., Midha, R., Cisterna, B. A., Grochmal, J., Shakhbazau, A., Hendriks, W. T., et al. (2011). Morphological evidence for a transport of ribosomes from Schwann cells to regenerating axons. Glia 59, 1529-1539. doi: 10.1002/glia.21196

Crino, P. B. (2011). mTOR: a pathogenic signaling pathway in developmental brain malformations. Trends Mol. Med. 17, 734-742. doi: 10.1016/j.molmed.2011.07.008

Garcia Moreno, L. M., Cimadevilla, J. M., Gonzalez Pardo, H., Zahonero, M. C., and Arias, J. L. (1997). NOR activity in hippocampal areas during the postnatal development and ageing. Mech. Ageing Dev. 97, 173-181. doi: 10.1016/S00476374(97)00054-7

Garcia-Moreno, L. M., Conejo, N. M., Pardo, H. G., Gomez, M., Martin, F. R., Alonso, M. J., et al. (2001). Hippocampal AgNOR activity after chronic alcohol consumption and alcohol deprivation in rats. Physiol. Behav. 72, 115-121. doi: 10.1016/S0031-9384(00)00408-X

Garthe, A., Behr, J., and Kempermann, G. (2009). Adult-generated hippocampal neurons allow the flexible use of spatially precise learning strategies. PLOS ONE 4:e5464. doi: 10.1371/journal.pone.0005464

Gentleman, R. C., Carey, V. J., Bates, D. M., Bolstad, B., Dettling, M., Dudoit, S., et al. (2004). Bioconductor: open software development for computational biology and bioinformatics. Genome Biol. 5, R80. doi: 10.1186/gb-2004-5-10-r80

Giese, K. P., Fedorov, N. B., Filipkowski, R. K., and Silva, A. J. (1998). Autophosphorylation at Thr286 of the alpha calcium-calmodulin kinase
II in LTP and learning. Science 279, 870-873. doi: 10.1126/science.279. 5352.870

Gomes, C., Smith, S. C., Youssef, M. N., Zheng, J. J., Hagg, T., and Hetman, M. (2011). RNA polymerase 1-driven transcription as a mediator of BDNF-induced neurite outgrowth. J. Biol. Chem. 286, 4357-4363. doi: 10.1074/jbc.M110.170134

Granneman, S., and Baserga, S. J. (2005). Crosstalk in gene expression: coupling and co-regulation of rDNA transcription, pre-ribosome assembly and pre-rRNA processing. Curr. Opin. Cell Biol. 17, 281-286. doi: 10.1016/j.ceb.2005.04.001

Grewal, S. S., Evans, J. R., and Edgar, B. A. (2007). Drosophila TIF-IA is required for ribosome synthesis and cell growth and is regulated by the TOR pathway. J. Cell Biol. 179, 1105-1113. doi: 10.1083/jcb.200709044

Hetman, M., and Pietrzak, M. (2012). Emerging roles of the neuronal nucleolus. Trends Neurosci. 35, 305-314. doi: 10.1016/j.tins.2012.01.002

Hoppe, S., Bierhoff, H., Cado, I., Weber, A., Tiebe, M., Grummt, I., et al. (2009). AMP-activated protein kinase adapts rRNA synthesis to cellular energy supply. Proc. Natl. Acad. Sci. U.S.A. 106, 17781-17786. doi: 10.1073/pnas. 0909873106

Iacono, D., O’Brien, R., Resnick, S. M., Zonderman, A. B., Pletnikova, O., Rudow, G., et al. (2008). Neuronal hypertrophy in asymptomatic Alzheimer disease. J. Neuropathol. Exp. Neurol. 67, 578-589. doi: 10.1097/NEN.0b013e31 81772794

Jordan, B. A., Fernholz, B. D., Khatri, L., and Ziff, E. B. (2007). Activity-dependent AIDA-1 nuclear signaling regulates nucleolar numbers and protein synthesis in neurons. Nat. Neurosci. 10, 427-435. doi: 10.1038/nn1867

Katz, L. M., Callaway, C. W., Kagan, V. E., and Kochanek, P. M. (1998). Electron spin resonance measure of brain antioxidant activity during ischemia/reperfusion. Neuroreport 9, 1587-1593. doi: 10.1097/00001756-199805110-00061

Kelleher, R. J. 3rd., and Bear, M. F. (2008). The autistic neuron: troubled translation? Cell 135, 401-406. doi: 10.1016/j.cell.2008.10.017

Kempermann, G., and Gage, F. H. (2002). Genetic determinants of adult hippocampal neurogenesis correlate with acquisition, but not probe trial performance, in the water maze task. Eur. J. Neurosci. 16, 129-136. doi: 10.1046/j.14609568.2002.02042.x

Kopp, K., Gasiorowski, J. Z., Chen, D., Gilmore, R., Norton, J. T., Wang, C., et al. (2007). Pol I transcription and pre-rRNA processing are coordinated in a transcription-dependent manner in mammalian cells. Mol. Biol. Cell 18, 394-403. doi: 10.1091/mbc.E06-03-0249

Kreiner, G., Bierhoff, H., Armentano, M., Rodriguez-Parkitna, J., Sowodniok, K., Naranjo, J. R., et al. (2013). A neuroprotective phase precedes striatal degeneration upon nucleolar stress. Cell Death Differ. 20, 1455-1464. doi: $10.1038 / \mathrm{cdd} .2013 .66$

Laplagne, D. A., Esposito, M. S., Piatti, V. C., Morgenstern, N. A., Zhao, C., van Praag, H., et al. (2006). Functional convergence of neurons generated in the developing and adult hippocampus. PLoS Biol. 4:e409. doi: 10.1371/journal.pbio.0040409

Laplante, M., and Sabatini, D. M. (2012). mTOR signaling in growth control and disease. Cell 149, 274-293. doi: 10.1016/j.cell.2012.03.017

Lledo, P. M., Alonso, M., and Grubb, M. S. (2006). Adult neurogenesis and functional plasticity in neuronal circuits. Nat. Rev. Neurosci. 7, 179-193. doi: $10.1038 / \mathrm{nrn} 1867$

Luzzati, F., De Marchis, S., Parlato, R., Gribaudo, S., Schutz, G., Fasolo, A., et al. (2011). New striatal neurons in a mouse model of progressive striatal degeneration are generated in both the subventricular zone and the striatal parenchyma. PLoS ONE 6:e25088. doi: 10.1371/journal.pone.0025088

Mantamadiotis, T., Lemberger, T., Bleckmann, S. C., Kern, H., Kretz, O., Martin Villalba, A., et al. (2002). Disruption of CREB function in brain leads to neurodegeneration. Nat. Genet. 31, 47-54. doi: 10.1038/ng882

Marquez-Lona, E. M., Tan, Z., and Schreiber, S. S. (2012). Nucleolar stress characterized by downregulation of nucleophosmin: a novel cause of neuronal degeneration. Biochem. Biophys. Res. Commun. 417, 514-520. doi: 10.1016/j.bbrc.2011.11.152

Mayer, C., Bierhoff, H., and Grummt, I. (2005). The nucleolus as a stress sensor: JNK2 inactivates the transcription factor TIF-IA and down-regulates rRNA synthesis. Genes Dev. 19, 933-941. doi: 10.1101/gad.333205

Mayer, C., Zhao, J., Yuan, X., and Grummt, I. (2004). mTOR-dependent activation of the transcription factor TIF-IA links rRNA synthesis to nutrient availability. Genes Dev. 18, 423-434. doi: 10.1101/gad.285504 
McGowan, P. O., Sasaki, A., Huang, T. C., Unterberger, A., Suderman, M., Ernst, C., et al. (2008). Promoter-wide hypermethylation of the ribosomal RNA gene promoter in the suicide brain. PLoS ONE 3:e2085. doi: 10.1371/journal.pone.0002085

Mendoza, M. C., Er, E. E., and Blenis, J. (2011). The Ras-ERK and PI3K-mTOR pathways: cross-talk and compensation. Trends Biochem. Sci. 36, 320-328. doi: 10.1016/j.tibs.2011.03.006

Moore, H. M., Bai, B., Matilainen, O., Colis, L., Peltonen, K., and Laiho, M. (2013). Proteasome activity influences UV-mediated subnuclear localization changes of NPM. PLoS ONE 8:e59096. doi: 10.1371/journal.pone.0059096

Murano, K., Okuwaki, M., Hisaoka, M., and Nagata, K. (2008). Transcription regulation of the rRNA gene by a multifunctional nucleolar protein, B23/nucleophosmin, through its histone chaperone activity. Mol. Cell. Biol. 28, 3114-3126. doi: 10.1128/MCB.02078-07

Parlato, R., and Kreiner, G. (2013). Nucleolar activity in neurodegenerative diseases: a missing piece of the puzzle? J. Mol. Med. (Berl.) 91, 541-547. doi: 10.1007/s00109-012-0981-1

Parlato, R., Kreiner, G., Erdmann, G., Rieker, C., Stotz, S., Savenkova, E., et al. (2008). Activation of an endogenous suicide response after perturbation of rRNA synthesis leads to neurodegeneration in mice. J. Neurosci. 28, 12759-12764. doi: 10.1523/JNEUROSCI.2439-08.2008

Piatti, V. C., Ewell, L. A., and Leutgeb, J. K. (2013). Neurogenesis in the dentate gyrus: carrying the message or dictating the tone. Front. Neurosci. 7:50. doi: 10.3389/fnins.2013.00050

Pietrzak, M., Rempala, G., Nelson, P. T., Zheng, J. J., and Hetman, M. (2011). Epigenetic silencing of nucleolar rRNA genes in Alzheimer's disease. PLoS ONE 6:e22585. doi: 10.1371/journal.pone.0022585

Reisel, D., Bannerman, D. M., Schmitt, W. B., Deacon, R. M., Flint, J., Borchardt, T., et al. (2002). Spatial memory dissociations in mice lacking GluR1. Nat. Neurosci. 5, 868-873. doi: 10.1038/nn910

Rieker, C., Engblom, D., Kreiner, G., Domanskyi, A., Schober, A., Stotz, S., et al. (2011). Nucleolar disruption in dopaminergic neurons leads to oxidative damage and parkinsonism through repression of mammalian target of rapamycin signaling. J. Neurosci. 31, 453-460. doi: 10.1523/JNEUROSCI.0590-10.2011

Rubbi, C. P., and Milner, J. (2003). Disruption of the nucleolus mediates stabilization of p53 in response to DNA damage and other stresses. EMBO J. 22, 6068-6077. doi: 10.1093/emboj/cdg579

Sanderson, D. J., Good, M. A., Seeburg, P. H., Sprengel, R., Rawlins, J. N., and Bannerman, D. M. (2008). The role of the GluR-A (GluR1) AMPA receptor subunit in learning and memory. Prog. Brain Res. 169, 159-178. doi: 10.1016/S0079-6123(07)00009-X

Santini, E., Huynh, T. N., MacAskill, A. F., Carter, A. G., Pierre, P., Ruggero, D., et al. (2013). Exaggerated translation causes synaptic and behavioural aberrations associated with autism. Nature 493, 411-415. doi: 10.1038/nature11782

Schmidt, B., Jacobson, T. K., and Markus, E. (2009). Hippocampal and striatal dependent navigation: sex differences are limited to acquisition. Horm. Behav. 56, 199-205. doi: 10.1016/j.yhbeh.2009.04.004 van Praag, H., Schinder, A. F., Christie, B. R., Toni, N., Palmer, T. D., and Gage, F. H. (2002). Functional neurogenesis in the adult hippocampus. Nature 415, 1030-1034. doi: 10.1038/4151030a

Widy-Tyszkiewicz, E., Scheel-Kruger, J., and Christensen, A. V. (1993). Spatial navigation learning in spontaneously hypertensive, renal hypertensive and normotensive Wistar rats. Behav. Brain Res. 54, 179-185. doi: 10.1016/01664328(93)90077-4

Wullschleger, S., Loewith, R., and Hall, M. N. (2006). TOR signaling in growth and metabolism. Cell 124, 471-484. doi: 10.1016/j.cell.2006.01.016

Yao, Z., Duan, S., Hou, D., Wang, W., Wang, G., Liu, Y., et al. (2010). B23 acts as a nucleolar stress sensor and promotes cell survival through its dynamic interaction with hnRNPU and hnRNPA1. Oncogene 29, 1821-1834. doi: 10.1038/onc.2009.473

Yuan, X., Zhou, Y., Casanova, E., Chai, M., Kiss, E., Grone, H. J., et al. (2005). Genetic inactivation of the transcription factor TIF-IA leads to nucleolar disruption, cell cycle arrest, and p53-mediated apoptosis. Mol. Cell 19, 77-87. doi: 10.1016/j.molcel.2005.05.023

Zamanillo, D., Sprengel, R., Hvalby, O., Jensen, V., Burnashev, N., Rozov, A., et al. (1999). Importance of AMPA receptors for hippocampal synaptic plasticity but not for spatial learning. Science 284, 1805-1811. doi: 10.1126/science.284.5421.1805

Zhao, J., Yuan, X., Frodin, M., and Grummt, I. (2003). ERK-dependent phosphorylation of the transcription initiation factor TIF-IA is required for RNA polymerase I transcription and cell growth. Mol. Cell 11, 405-413. doi: 10.1016/S1097-2765(03)00036-4

Conflict of Interest Statement: The authors declare that the research was conducted in the absence of any commercial or financial relationships that could be construed as a potential conflict of interest.

Received: 27 May 2013; accepted: 19 October 2013; published online: 11 November 2013.

Citation: Kiryk A, Sowodniok K, Kreiner G, Rodriguez-Parkitna J, Sönmez A, Górkiewicz T, Bierhoff H, Wawrzyniak M, Janusz AK, Liss B, Konopka W, Schütz $G$, Kaczmarek L and Parlato R (2013) Impaired rRNA synthesis triggers homeostatic responses in hippocampal neurons. Front. Cell. Neurosci. 7:207. doi: 10.3389/fncel. 2013.00207

This article was submitted to the journal Frontiers in Cellular Neuroscience.

Copyright (c) 2013 Kiryk, Sowodniok, Kreiner, Rodriguez-Parkitna, Sönmez, Górkiewicz, Bierhoff, Wawrzyniak, Janusz, Liss, Konopka, Schütz, Kaczmarek and Parlato. This is an open-access article distributed under the terms of the Creative Commons Attribution License (CC BY). The use, distribution or reproduction in other forums is permitted, provided the original author(s) or licensor are credited and that the original publication in this journal is cited, in accordance with accepted academic practice. No use, distribution or reproduction is permitted which does not comply with these terms. 